\title{
THE TWO-PHASE FRACTIONAL OBSTACLE PROBLEM
}

\author{
MARK ALLEN, ERIK LINDGREN, AND ARSHAK PETROSYAN
}

AbSTRACT. We study minimizers of the functional

$$
\int_{B_{1}^{+}}|\nabla u|^{2} x_{n}^{a} \mathrm{~d} x+2 \int_{B_{1}^{\prime}}\left(\lambda_{+} u^{+}+\lambda_{-} u^{-}\right) \mathrm{d} x^{\prime}
$$

for $a \in(-1,1)$. The problem arises in connection with heat flow with control on the boundary. It can also be seen as a non-local analogue of the, by now well studied, two-phase obstacle problem. Moreover, when $u$ does not change signs this is equivalent to the fractional obstacle problem. Our main results are the optimal regularity of the minimizer and the separation of the two free boundaries $\Gamma^{+}=\partial^{\prime}\{u(\cdot, 0)>0\}$ and $\Gamma^{-}=\partial^{\prime}\{u(\cdot, 0)<0\}$ when $a \geq 0$.

\section{INTRODUCTION AND MOTIVATION}

1.1. The problem. The main purpose of this paper is to study the minimizers of the energy functional

$$
J_{a}(u)=\int_{D^{+}}|\nabla u|^{2} x_{n}^{a} \mathrm{~d} x+2 \int_{D^{\prime}}\left(\lambda_{+} u^{+}+\lambda_{-} u^{-}\right) \mathrm{d} x^{\prime},
$$

where $D$ is a bounded domain in $\mathbb{R}^{n}$,

$$
D^{+}:=D \cap\left\{x_{n}>0\right\}, \quad D^{\prime}:=D \cap\left\{x_{n}=0\right\},
$$

$a \in(-1,1), \lambda_{ \pm}>0$, and $u^{ \pm}=\max \{ \pm u, 0\}$. More precisely, we look at the functions $u$ in the weighted Sobolev space $W^{1,2}\left(D^{+}, x_{n}^{a}\right)$, with prescribed boundary values

$$
u=g \quad \text { on }(\partial D)^{+}:=\partial D \cap\left\{x_{n}>0\right\},
$$

in the sense of traces. This leads to a two-phase problem on $D^{\prime}$ if we identify the regions

$$
\Omega^{+}=\Omega_{u}^{+}:=\{u>0\} \cap D^{\prime}, \quad \Omega^{-}=\Omega_{u}^{-}:=\{u<0\} \cap D^{\prime}
$$

as the phases of the minimizer $u$. We call their boundaries in $D^{\prime}$

$$
\Gamma^{+}=\Gamma_{u}^{+}:=\partial^{\prime} \Omega^{+} \cap D^{\prime}, \quad \Gamma^{-}=\Gamma_{u}^{-}:=\partial^{\prime} \Omega^{-} \cap D^{\prime}
$$

free boundaries, since they are apriori unknown $\left(\partial^{\prime}\right.$ here stands for the boundary in $\mathbb{R}^{n-1}$ ). The free boundaries of this type are known as thin free boundaries in the literature, since they are formally of co-dimension two. Particular questions of interest are the regularity of the minimizers and the regularity and structure of their free boundaries.

2010 Mathematics Subject Classification. Primary 35R35. Secondary 35B65, 35J70.

Key words and phrases. Thin obstacle problem, fractional obstacle problem, two-phase free boundary problem, separation of phases, fractional Laplacian, Almgen's frequency formula, Weisstype monotonicity formula, Alt-Caffarelli-Friedman monotoncity formula.

M.A. and A.P. are partially supported by NSF grant DMS-1101139. E.L. thanks the Royal Swedish Academy of Sciences for partial support. 
The problem above is strongly related to the so-called obstacle problem for fractional Laplacian $\left(-\Delta_{x^{\prime}}\right)^{s}, 0<s<1$, or more precisely its localized version. The latter problem consists of minimizing the energy functional

$$
E_{a}(v)=\int_{D^{+}}|\nabla v|^{2} x_{n}^{a} \mathrm{~d} x, \quad a=1-2 s
$$

among all functions $v \in W^{1,2}\left(D^{+}, x_{n}^{a}\right)$ satisfying

$$
\begin{array}{ll}
v=h & \text { on }(\partial D)^{+}, \\
v \geq \phi & \text { on } D^{\prime} .
\end{array}
$$

The function $\phi$ above is know as the thin obstacle. In the particular case of $a=0$, this problem is the scalar version of the Signorini problem, also know as the thin (or boundary) obstacle problem. The free boundary in this problem is

$$
\Gamma_{v}=\partial^{\prime}\{v(\cdot, 0)>\phi\} \cap D^{\prime} .
$$

There has been a surge of interest in such problems in recent years, because of the development of new tools such as the Caffarelli-Silvestre extension, Almgren-type monotonicity formulas, etc, that allowed significant advances in the study of the regularity properties of the free boundaries; see [AC04, Sil07, ACS08, CSS08, GP09.

The exact connection between the minimizers of (1.1)-(1.2) and (1.3)-(1.5) is given in the following (relatively straightforward) proposition.

Proposition 1.1. Let $u$ be a minimizer of (1.1)-(1.2) such that $u \geq 0$ on $D^{\prime}$. Then $v(x)=u(x)-\frac{\lambda_{+}}{1-a} x_{n}^{1-a}$ is a minimizer of (1.3) with $h(x)=g(x)-\frac{\lambda_{+}}{1-a} x_{n}^{1-a}$ and $\phi=0$.

Proof. The proof is an immediate corollary of the following computation:

$$
\begin{aligned}
E_{a}(v) & =\int_{D^{+}}\left|\nabla u-\lambda_{+} x_{n}^{-a} e_{n}\right|^{2} x_{n}^{a} \\
& =\int_{D^{+}}\left(|\nabla u|^{2} x_{n}^{a}+\lambda_{+}^{2} x_{n}^{-a}-2 \lambda_{+} \partial_{x_{n-1}} u\right) \\
& =\int_{D^{+}}|\nabla u|^{2} x_{n}^{a}+2 \int_{D^{\prime}}\left(\lambda_{+} u^{+}\right)+C\left(D^{+}, g\right) \\
& =J_{a}(u)+C\left(D^{+}, g\right) .
\end{aligned}
$$

In particular, when $u \geq 0$ we see that $u\left(x^{\prime}, 0\right) \equiv v\left(x^{\prime}, 0\right)$ and consequently

$$
\Gamma_{u}^{+}=\Gamma_{v},
$$

reducing the study of the free boundary in (1.1)-(1.2) to that in the fractional obstacle problem (1.3)-(1.5). For the reasons above, we call the minimization problem (1.1)-(1.2) the two-phase fractional obstacle problem. When $a=0$ we also call it the two-phase thin obstacle problem.

The two-phase fractional obstacle problem can also be viewed as a nonlocal version of the so-called two-phase obstacle problem (also known as the two-phase membrane problem)

$$
\Delta u=\lambda_{+} \chi_{\{u>0\}}-\lambda_{-} \chi_{\{u<0\}} \quad \text { in } D .
$$


Solutions of (1.6) can be obtained by minimizing the the energy functional

$$
\tilde{J}(u)=\int_{D}\left(|\nabla u|^{2}+2 \lambda_{+} u^{+}+2 \lambda_{-} u^{-}\right) \mathrm{d} x .
$$

This problem has been studied in detail in a series of papers Wei01, Ura01, SUW04, SW06, SUW07. In particular, it is known that the minimizers are $C^{1,1}$-regular and that the free boundaries $\tilde{\Gamma}^{ \pm}:=\partial\{u>0\} \cap D$ are graphs of $C^{1}$ functions near twophase points (i.e. points on $\tilde{\Gamma}^{+} \cap \tilde{\Gamma}^{-}$). Away from two-phase points, the problem is locally equivalent to the classical obstacle problem, which is extensively studied in the literature. We also refer to the book [PSU12] for the introduction to this and other obstacle-type problems.

1.2. Main results. Our first result concerns the regularity of the minimizers.

Theorem 1.2 (Optimal regularity). Let $u$ be a minimizer of (1.1). Then for any $K \Subset D^{+} \cup D^{\prime}$ we have

$$
\begin{array}{ll}
u \in C^{0,1-a}(K), & \text { if } a \geq 0 \\
u \in C^{1,-a}(K), & \text { if } a<0
\end{array}
$$

with bounds on their respective norms, depending only on $K, D, a, \lambda_{ \pm}, n$, and $\|u\|_{L^{2}\left(D^{+}\right)}$.

Note that this regularity is sharp, because of the explicit example

$$
u(x)=\frac{\lambda_{+}}{(1-a)} x_{n}^{1-a},
$$

see Proposition 1.1

As a by-product we also obtain Theorem 6.4, which, by the Caffarelli-Silvestre extension, provides sharp regularity estimates for solutions of

$$
(-\Delta)^{s} u=f \in L^{\infty},
$$

if $s=(1-a) / 2 \neq 1 / 2$.

Our second result is about the structure of the free boundary, which effectively reduces the problem to the (one-phase) fractional obstacle problem in the case $a \geq 0$.

Theorem 1.3 (Structure of the free boundary). Let $a \geq 0$ and let $u$ be a minimizer of (1.1). Then

$$
\Gamma^{+} \cap \Gamma^{-}=\emptyset .
$$

Thus, by Proposition 1.1, the study of the free boundary is reduced to the fractional obstacle problem.

\subsection{Structure of the paper.}

- In Section 2, we describe the notation used throughout the paper.

- Section 3 contains a couple of applications that motivated our study.

- In Section 4 we collected some results on $a$-harmonic functions: interior estimates, Liouville-type theorem and Almgren's frequency formula, as well as some preliminary results on our problem: energy inequality, local boundedness, Weisstype monotonicity formula (the proof of the latter is given in Appendix). It also contains the Alt-Caffarelli-Friedman monotonicity formula to be used in the case $a=0$. 
- In Section 5 we prove some preliminary regularity on the minimizers: namely $C^{0,(1-a) / 2}$, when $a \geq 0$, and $C_{x^{\prime}}^{1, \alpha}$ for $\alpha<-a$, when $a<0$.

- Section 6 contains the proof of Theorem 1.2 We first show the optimal growth rate of the minimizers from the free boundary (Theorem 6.1) which then implies the optimal regularity (Theorem 6.3).

- In Section 7 we prove the nondegeneracy of each phase near their respective free boundaries.

- In Section 8 we study the blowups, by showing first that they are homogeneous of degree $(1-a)$ and then classifying all such minimizers for $a \geq 0$.

- Finally, in Section 9 we prove Theorem 1.3. In fact, we prove its stronger version (Theorem 9.1) that the free boundaries $\Gamma^{+}$and $\Gamma^{-}$are uniformly separated when $a \geq 0$. We conclude the paper with an example of a solution when $a<0$ where $\Gamma^{+}$and $\Gamma^{-}$have a common part with vanishing thin gradient $\left|\nabla_{x^{\prime}} u\right|$.

\section{Notation}

Throughout the paper we will be using the following notation:

- We denote a point $x \in \mathbb{R}^{n}$ by $\left(x^{\prime}, x_{n}\right)$ where $x^{\prime}=\left(x_{1}, \ldots, x_{n-1}\right) \in \mathbb{R}^{n-1}$. Moreover, in certain cases we will identify $x^{\prime} \in \mathbb{R}^{n-1}$ with $\left(x^{\prime}, 0\right) \in \mathbb{R}^{n-1} \times\{0\} \subset \mathbb{R}^{n}$. We will refer to $\mathbb{R}^{n-1}=\mathbb{R}^{n-1} \times\{0\}$ as the thin space.

$-\nabla u=\left(\partial_{x_{1}} u, \ldots, \partial_{x_{n-1}} u, \partial_{x_{n}} u\right)$ : the full gradient.

- $\nabla_{x^{\prime}} u=\left(\partial_{x_{1}} u, \ldots, \partial_{x_{n-1}} u\right)$ : the gradient in the thin space, or the thin gradient.

- $u_{x_{i}}$ : alternative notation for the partial derivative $\partial_{x_{i}} u, i=1, \ldots, n$.

- For a set $E \subset \mathbb{R}^{n}$ we define

$$
E^{\prime}:=E \cap\left(\mathbb{R}^{n-1} \times\{0\}\right)
$$

- For $E \subset \mathbb{R}^{n-1}=\mathbb{R}^{n-1} \times\{0\}, \partial^{\prime} E$ is the boundary in the topology of $\mathbb{R}^{n-1}$.

- For a function $u$, we define $u^{+}=\max \{u, 0\}$ and $u^{-}=\max \{-u, 0\}$, the positive and negative parts of $u$ so that, $u=u^{+}-u^{-}$.

- We call $\Gamma^{+}$and $\Gamma^{-}$the free boundaries where

$$
\Gamma^{+}=\partial^{\prime}\{u(\cdot, 0)>0\} \text { and } \Gamma^{-}=\partial^{\prime}\{u(\cdot, 0)<0\}
$$

- We will for $x_{0} \in \Gamma^{+} \cup \Gamma^{-}$, denote by $u_{r, x_{0}}$, the rescaled function

$$
\frac{u\left(r x+x_{0}\right)}{r^{1-a}} \text {. }
$$

If there is a subsequence $u_{r_{j}, x_{0}}$, where $r_{j} \rightarrow 0$, converging locally uniformly to a function $u_{0, x_{0}}$ we will say that $u_{0, x_{0}}$ is a blow-up of $u$ at the point $x_{0}$.

- $L^{2}\left(D^{+}, x_{n}^{a}\right)$ [respectively $\left.L^{2}\left(D,\left|x_{n}\right|^{a}\right)\right]$, for $|a|<1$, is the weighted Lebesgue space with the square of the norm given by

$$
\int_{D^{+}}|f(x)|^{2} x_{n}^{a} \mathrm{~d} x \quad\left[\text { respectively } \int_{D}|f(x)|^{2}\left|x_{n}\right|^{a} \mathrm{~d} x\right] .
$$

- $W^{1,2}\left(D^{+}, x_{n}^{a}\right)\left[\right.$ respectively $\left.W^{1,2}\left(D,\left|x_{n}\right|^{a}\right)\right]$, for $|a|<1$, is the weighted Sobolev space of functions $u \in L^{2}\left(D^{+}, x_{n}^{a}\right)$ [respectively $L^{2}\left(D,\left|x_{n}\right|^{a}\right)$ ] such that the distributional derivatives $\partial_{x_{i}} u, i=1, \ldots, n$, are also in $L^{2}\left(D^{+}, x_{n}^{a}\right)$ [respectively $\left.L^{2}\left(D,\left|x_{n}\right|^{a}\right)\right]$. For some of the basic results for these spaces such as the Sobolev embedding and Poincaré inequalities, we refer to [FKS82. See also [Kil97.

It is known that if there is a bi-Lipschitz transformation that will take $\left(D, D^{\prime}\right)$ to $\left(B_{1}, B_{1}^{\prime}\right)$ then functions in $W^{1,2}\left(D^{+}, x_{n}^{a}\right)$ or $\left.W^{1,2}\left(D,\left|x_{n}\right|^{a}\right)\right]$ have traces in 
$L^{2}\left(D^{\prime}\right)$ and $L^{2}\left((\partial D)^{+}, x_{n}^{a}\right)\left[\right.$ or $\left.L^{2}\left(\partial D,\left|x_{n}\right|^{a}\right)\right]$. Furthermore, the corresponding trace operators are compact. See [Nek93] for the first trace operator. Compactness of the second one follows from the approximation by Lipschitz functions as indicated in Remark 6 in [Kil97, similarly to the proof of Theorem 8 there.

- We say that $u \in W^{1,2}\left(D,\left|x_{n}\right|^{a}\right)$ is a-harmonic in an open set $D \subset \mathbb{R}^{n}$ if it is a weak solution of

$$
L_{a} u:=\operatorname{div}\left(\left|x_{n}\right|^{a} \nabla u\right)=0 .
$$

Equivalently, $a$-harmonic functions are the minimizers of the weighted Dirichlet integral

$$
E_{a}(u):=\int_{D}|\nabla u|^{2}\left|x_{n}\right|^{a} \mathrm{~d} x,
$$

among all functions with the same trace on $\partial D$.

- For $u \in W^{1,2}\left(D,\left|x_{n}\right|^{a}\right)$, by an a-harmonic replacement of $u$ on $U \Subset D$ we understand a function $v$ coinciding with $u$ on $D \backslash U, a$-harmonic in $U$, with the same boundary values on $\partial U$ as $u$ in the sense $v-u \in W_{0}^{1,2}\left(U,\left|x_{n}\right|^{a}\right)$.

- Very often we will use the following convention for integrals. If the measure of integration is not indicated then it is either the standard Lebesgue measure $\mathrm{d} x$ in $\mathbb{R}^{n}$, or $(n-1)$-dimensional Hausdorff measure $\mathrm{d} \mathcal{H}^{n-1}$ (also denoted $\mathrm{d} x^{\prime}$ when restricted to $\left.\mathbb{R}^{n-1}\right)$.

\section{Motivation}

In this section we describe some applications that motivated us to study the problem (1.1)-(1.2), in addition to those indicated in the introduction.

3.1. Temperature control problems. One of the applications of the two-phase obstacle problem (1.6) is the temperature control problem through the interior, see Duvaut-Lions DL76, Chapter I, §2.3.2]. More specifically, suppose that we want to maintain the temperature $u(x)$ in the domain $D$ close to the range $\left(\theta_{-}, \theta_{+}\right)$, where $\theta_{-}(x) \leq \theta_{+}(x)$ are two given functions in $D$. For that purpose we have an array of cooling/heating devices that are distributed evenly over the domain $D$. The devices are assumed to be of limited power, with their generated heat flux $-f$ in the range $\left[-\lambda_{-}, \lambda_{+}\right], \lambda_{ \pm}>0$. The device will generate no heat if $u(x) \in\left[\theta_{-}(x), \theta_{+}(x)\right]$, however, outside that range the corrective heat flux $F$ will be injected according to the following rule:

$$
F=F(u)= \begin{cases}\min \left\{k_{+}\left(u-\theta_{+}\right), \lambda_{+}\right\}, & u>\theta_{+}, \\ \max \left\{k_{-}\left(u-\theta_{-}\right),-\lambda_{-}\right\}, & u<\theta_{-},\end{cases}
$$

where $k_{ \pm} \geq 0$. In the equilibrium state, the temperature distribution will satisfy

$$
\Delta u=F(u) \text { in } D .
$$

Assuming $\theta_{ \pm}=0$ and $k_{ \pm}=+\infty$, the equation becomes

$$
\Delta u=\lambda_{+} \chi_{\{u>0\}}-\lambda_{-} \chi_{\{u<0\}} \text { in } D,
$$

which is nothing but the two-phase obstacle problem.

Suppose now that we want to regulate the temperature in $D^{+}=D \cap\left\{x_{n}>0\right\}$ with the heating/cooling devices that are evenly distributed along the "wall" $D^{\prime}=$ $D \cap\left\{x_{n}=0\right\}$. Then in the equilibrium position, the temperature will satisfy

$$
\Delta u=0 \quad \text { in } D^{+}, \quad \partial_{x_{n}} u=F(u) \text { on } D^{\prime} .
$$


In the limiting case $\theta_{ \pm}=0$ and $k_{ \pm}=\infty$ this transforms into the variational inequality

$$
\Delta u=0 \quad \text { in } D, \quad \partial_{x_{n}} u \in \bar{F}(u) \quad \text { on } D^{\prime},
$$

where $\bar{F}$ is the maximal monotone graph

$$
\bar{F}(u)= \begin{cases}\left\{\lambda_{+}\right\}, & u>0, \\ {\left[-\lambda_{-}, \lambda_{+}\right],} & u=0, \\ \left\{-\lambda_{-}\right\}, & u<0 .\end{cases}
$$

Equivalently, the solutions of this variational inequality can be obtained by minimizing the energy functional

$$
J_{0}(u)=\int_{D^{+}}|\nabla u|^{2} \mathrm{~d} x+2 \int_{D^{\prime}}\left(\lambda_{+} u^{+}+\lambda_{-} u^{-}\right) \mathrm{d} x^{\prime},
$$

subject to a Dirichlet boundary condition on $(\partial D)^{+}$(cf. Lemma 4.1). This is a particular case $(a=0)$ of the problem that we intend to study in this paper.

3.2. Two-phase problem for fractional Laplacian. Suppose now that $\Sigma$ is a bounded open set in $\mathbb{R}^{n-1}, 0<s<1$, and we consider the minimizers of the energy functional

$$
j_{s}(v)=c_{n, s} \int_{\mathbb{R}^{n-1}} \int_{\mathbb{R}^{n-1}} \frac{\left(v\left(x^{\prime}\right)-v\left(y^{\prime}\right)\right)^{2}}{\left|x^{\prime}-y^{\prime}\right|^{n-1+2 s}}+2 \int_{\mathbb{R}^{n-1}}\left(\lambda_{+} u^{+}+\lambda_{-} u^{-}\right),
$$

among all function such that $v=g$ on $\mathbb{R}^{n-1} \backslash \Sigma$. Here $c_{n, s}>0$ is a normalization constant. Then it is easy to see that the minimizers will satisfy the variational inequality

$$
-\left(-\Delta_{x^{\prime}}\right)^{s} v \in \bar{F}(v) \text { on } \Sigma \text {, }
$$

with the maximal monotone graph $\bar{F}$ in (3.1). To see the connection with the twophase thin obstacle problem, we use the Caffarelli-Silvestre extension CS07 for $v$. Namely, let $u$ be a function $\mathbb{R}_{+}^{n}$ that satisfies

$$
\begin{aligned}
L_{a} u:=\operatorname{div}\left(x_{n}^{a} \nabla u\right)=0 & \text { in } \mathbb{R}_{+}^{n} \\
u\left(x^{\prime}, 0\right)=v\left(x^{\prime}\right) & \text { for } x^{\prime} \in \mathbb{R}^{n-1},
\end{aligned}
$$

where $a=1-2 s \in(-1,1)$. (The initial condition is understood in the boundary trace sense.) Then we can recover the fractional Laplacian of $v$ on $\mathbb{R}^{n-1}$ by

$$
\left(-\Delta_{x^{\prime}}\right)^{s} v\left(x^{\prime}\right)=C_{n, s} \lim _{x_{n} \rightarrow 0+} x_{n}^{a} \partial_{x_{n}} u\left(x^{\prime}, x_{n}\right),
$$

in the weak sense. Suppose now that there is a smooth domain $D$ in $\mathbb{R}^{n}$ is such that $\Sigma=D^{\prime}=D \cap\left\{x_{n}=0\right\}$. Then, using this extension we see that the same $u$ can be recovered by minimizing the energy functional $J_{a}$ among all function $u$ with fixed Dirichlet condition on $(\partial D)^{+}$, which is exactly the problem we stated in the beginning of the introduction. 


\section{Preliminaries}

In this section we present certain results about the minimizers of (1.1) and $a$ harmonic functions that we will utilize later on.

We start with a remark that the results that we are interested in are local in nature and therefore without loss of generality we may assume that $D=B_{1}$ in (1.1). Moreover, it will also be convenient to extend the minimizers $u$ from $B_{1}^{+}$to the full ball $B_{1}$ by even symmetry

$$
u\left(x^{\prime},-x_{n}\right)=u\left(x^{\prime}, x_{n}\right) .
$$

Then such $u$ is in $W^{1,2}\left(B_{1},\left|x_{n}\right|^{a}\right)$ and is the minimizer of the functional

$$
J_{a}(u)=\frac{1}{2} \int_{B_{1}}|\nabla u|^{2}\left|x_{n}\right|^{a} \mathrm{~d} x+2 \int_{B_{1}^{\prime}}\left(\lambda_{+} u^{+}+\lambda_{-} u^{-}\right) \mathrm{d} x^{\prime}
$$

subject to the boundary condition

$$
u=g \quad \text { on } \partial B_{1}
$$

in the sense of traces, where $g \in L^{2}\left(\partial B_{1},\left|x_{n}\right|^{a}\right)$ is also evenly extended to $\partial B_{1}$ from $\left(\partial B_{1}\right)^{+}$.

The existence of minimizers of (4.1) -4.2), follows by the direct method in the calculus of variation, since $J_{a}$ is continuous on $W^{1,2}\left(B_{1},\left|x_{n}\right|^{a}\right)$. The uniqueness follows from the strict convexity of $J_{a}$ on the closed convex subset of functions in $W^{1,2}\left(B_{1},\left|x_{n}\right|^{a}\right)$ satisfying (4.2). The next lemma identifies the variational inequality satisfied by the minimizers.

Lemma 4.1 (Variational inequality). Let $u$ be a minimizer of (4.1). Then for $x^{\prime} \in B_{1}^{\prime}$ we have

$$
-\lim _{x_{n} \rightarrow 0^{+}} x_{n}^{a} u_{x_{n}}\left(x^{\prime}, x_{n}\right) \in \bar{F}(u)= \begin{cases}\left\{\lambda_{+}\right\} & u>0 \\ {\left[-\lambda_{-}, \lambda_{+}\right],} & u=0 \\ \left\{-\lambda_{-}\right\}, & u<0\end{cases}
$$

in the sense that there exists a measurable function $f$ in $B_{1}^{\prime}$ such that

$$
\begin{array}{r}
f\left(x^{\prime}\right) \in \bar{F}\left(u\left(x^{\prime}, 0\right)\right) \quad \text { for a.e. } x^{\prime} \in B_{1}^{\prime}, \text { and } \\
-\int_{B_{1}^{+}}\left|x_{n}\right|^{a} \nabla u \nabla \psi \mathrm{d} x=\int_{B_{1}^{\prime}} f \psi \mathrm{d} x^{\prime} \quad \text { for any } \psi \in C_{0}^{\infty}\left(B_{1}\right) .
\end{array}
$$

Remark 4.2. Taking $\psi\left(x^{\prime}, x_{n}\right)=\gamma\left(x^{\prime}\right) \eta\left(x_{n}\right)$ in (4.4) with $\gamma \in C_{0}^{\infty}\left(B_{1}^{\prime}\right)$ and $\eta \in$ $C_{0}^{\infty}([0,1)), \eta \equiv 1$ near 0 , we obtain that the convergence in (4.3) can be understood also in the sense of distributions

$$
\lim _{x_{n} \rightarrow 0+} \int_{B_{1}^{\prime}} x_{n}^{a} u_{x_{n}}\left(x^{\prime}, x_{n}\right) \gamma\left(x^{\prime}\right) \mathrm{d} x^{\prime}=\int_{B_{1}^{\prime}} f \gamma \mathrm{d} x^{\prime} .
$$

Proof. In fact, we will prove something more general: if $u$ is a minimizer of the functional

$$
J(u)=\int_{B_{1}^{+}}|\nabla u|^{2} x_{n}^{a}+2 \int_{B_{1}^{\prime}} \Psi(u),
$$

with a fixed Dirichlet data on $\left(\partial B_{1}\right)^{+}$, where $\Psi(s)$ is a convex function of $s \in \mathbb{R}$, then

$$
\int_{B_{1}^{\prime}} \Psi^{\prime}(u-) \psi \leq-\int_{B_{1}^{+}}\left|x_{n}\right|^{a} \nabla u \nabla \psi \leq \int_{B_{1}^{\prime}} \Psi^{\prime}(u+) \psi
$$


for any nonnegative $\psi \in C_{0}^{\infty}\left(B_{1}\right)$. Indeed, for such $\psi$ consider a competing function $u+\epsilon \psi$. From the minimality of $u$ we then have

$$
\begin{aligned}
0 \leq \frac{J(u+\epsilon \psi)-J(u)}{\epsilon}=2 & \int_{B_{1}^{+}} x_{n}^{a} \nabla u \nabla \psi+\epsilon \int_{B_{1}^{+}}|\nabla \psi|^{2} x_{n}^{a} \\
& +\int_{B_{1}^{\prime}} \frac{\Psi(u+\epsilon \psi)-\Psi(u)}{\epsilon} .
\end{aligned}
$$

Now, noting that $(\Psi(u+\epsilon \psi)-\Psi(u)) / \epsilon$ is monotone in $\epsilon>0$ and converges to $\Psi^{\prime}(u+) \psi$, by the monotone convergence theorem we will arrive at

$$
-\int_{B_{1}^{+}}\left|x_{n}\right|^{a} \nabla u \nabla \psi \leq \int_{B_{1}^{\prime}} \Psi^{\prime}(u+) \psi .
$$

The inequality from below is proved similarly.

The minimizers of (4.1) also enjoy the following comparison principle.

Lemma 4.3 (Comparison principle). Let $u, v$ be two minimizers of the functional (4.1) with $\left.u\right|_{\partial B_{1}} \leq\left. v\right|_{\partial B_{1}}$. Then $u \leq v$ in $B_{1}$.

Proof. If we define $\bar{w}:=\max \{u, v\}$ and $\underline{w}:=\min \{u, v\}$, it is straightforward to verify that

$$
J(\bar{w})+J(\underline{w})=J(u)+J(v)
$$

Since the functional is strictly convex on functions with the same boundary data, minimizers are unique. Since $\left.\bar{w}\right|_{\partial B_{1}}=\left.v\right|_{\partial B_{1}}$ and $\left.\underline{w}\right|_{\partial B_{1}}=\left.u\right|_{\partial B_{1}}$, we may therefore conclude that $u=\underline{w}, v=\bar{w}$, readily implying that $u \leq v$ in $B_{1}$.

Corollary 4.4. If the boundary data are symmetric about the line $\left(0, \ldots, 0, x_{n}\right)$, then the minimizer is symmetric about the line $\left(0, \ldots, 0, x_{n}\right)$.

Proof. Any rotation will be a minimizer, and minimizers are unique.

Lemma 4.5. Let $u$ be a minimizer of (4.1). Then $u^{ \pm}$are a-subharmonic functions, i.e., $L_{a} u^{ \pm} \geq 0$ in the weak sense

$$
\int_{B_{1}} \nabla\left(u^{ \pm}\right) \nabla \psi\left|x_{n}\right|^{a} \leq 0
$$

for any nonnegative $\psi \in W_{0}^{1,2}\left(B_{1},\left|x_{n}\right|^{a}\right)$.

Proof. For the nonnegative test function $\psi \in W_{0}^{1,2}\left(B_{1},\left|x_{n}\right|^{a}\right)$ and small $\epsilon>0$, let

$$
u_{\epsilon}=(u-\epsilon \psi)^{+}-u_{-} \text {. }
$$

Since $u=u_{\epsilon}$ on $\partial B_{1}$, from minimality of $u$ we must have $J_{a}(u) \leq J_{a}\left(u_{\epsilon}\right)$. Noticing that, $u_{\epsilon}^{+}=(u-\epsilon \psi)^{+} \leq u^{+}$and $u_{\epsilon}^{-}=u^{-}$we therefore have

$$
\begin{aligned}
\int_{B_{1} \cap\{u>0\}}|\nabla u|^{2}\left|x_{n}\right|^{a} & \leq \int_{B_{1} \cap\{u>\epsilon \psi\}}|\nabla u-\epsilon \nabla \psi|^{2}\left|x_{n}\right|^{a} \\
& \leq \int_{B_{1} \cap\{u>0\}}|\nabla u-\epsilon \nabla \psi|^{2}\left|x_{n}\right|^{a} .
\end{aligned}
$$

This readily implies that

$$
\int_{B_{1}} \nabla\left(u^{+}\right) \nabla \psi\left|x_{n}\right|^{a}=\int_{B_{1} \cap\{u>0\}} \nabla u \nabla \psi\left|x_{n}\right|^{a} \leq 0 .
$$


This proves $a$-subharmonicity of $u^{+}$. Arguing similarly, we establish the same fact also for $u^{-}$.

Corollary 4.6 (Energy inequality). Let $u$ be a minimizer of (4.1). Then we have the following inequality

$$
\int_{B_{r}}|\nabla u|^{2}\left|x_{n}\right|^{a} \leq \frac{C}{r^{2}} \int_{B_{2 r}} u^{2}\left|x_{n}\right|^{a} .
$$

Proof. Since $u^{ \pm}$are nonnegative and $a$-subharmonic, the standard proof of the Caccioppoli inequality (with test functions $u^{ \pm} \eta^{2}$ ) applies and gives

$$
\int_{B_{r}}\left|\nabla u_{ \pm}\right|^{2}\left|x_{n}\right|^{a} \leq \frac{C_{n}}{r^{2}} \int_{B_{2 r}} u_{ \pm}^{2}\left|x_{n}\right|^{a} .
$$

Combining these two inequalities, we complete the proof.

Corollary 4.7 (Local boundedness). Let $u$ be a minimizer (4.1). Then

$$
\|u\|_{L^{\infty}\left(B_{1 / 2}\right)} \leq C(n, a)\|u\|_{L^{2}\left(B_{1},\left|x_{n}\right|^{a}\right)}
$$

Proof. This follows from $L^{\infty}-L^{2}$ estimates for $a$-subharmonic functions $u^{ \pm}$, see FKS82.

For our further study, we will need some properties of $a$-harmonic functions. We start with the observation that Corollaries 4.6 and 4.7 are applicable also to $a$-harmonic functions (e.g., take $\lambda_{ \pm}=0$ in the proofs above). Besides, since $\left|x_{n}\right|^{a}$ is an $A_{2}$ weight, we have the following result from [FKS82.

Lemma 4.8 (Hölder continuity). Let $u$ be a-harmonic in $B_{1}$. Then for some $\alpha>0$, $u \in C^{0, \alpha}\left(B_{1 / 2}\right)$ and

$$
\|u\|_{C^{0, \alpha}\left(B_{1 / 2}\right)} \leq C(n, a, \alpha)\|u\|_{L^{2}\left(B_{1}\right)} .
$$

Next, we the following derivative estimates in $x^{\prime}$ directions (see CSS08, Corollary 2.5]).

Lemma 4.9 (Interior estimates). Let $u$ be a-harmonic in a ball $B_{r}(x)$. Then for any positive integer $k$

$$
\sup _{B_{r / 2}(x)}\left|D_{x^{\prime}}^{k} u\right| \leq \frac{C(n, a, k)}{r^{k}} \underset{B_{r}(x)}{\operatorname{osc}} u
$$

Note that because of the weight $\left|x_{n}\right|^{a}$, similar estimates for the derivatives in $x_{n}$ must be weighted accordingly.

Further, at several points we will need the following result [CSS08, Lemma 2.7].

Lemma 4.10 (Liouville-type theorem). Let $u$ be a-harmonic in $\mathbb{R}^{n}$, even with respect to $x_{n}$ and

$$
|u(x)| \leq C\left(1+|x|^{k}\right),
$$

for some $C, k>0$. Then $u$ is a polynomial.

We next state an Almgren-type frequency formula, which first appeared in CS07. Theorem 6.1]: 
Lemma 4.11 (Almgren-type frequency formula). Let $u$ be a-harmonic in $B_{1}$. Then the function

$$
N(r)=N(u, r)=\frac{r \int_{B_{r}}|\nabla u|^{2}\left|x_{n}\right|^{a}}{\int_{\partial B_{r}} u^{2}\left|x_{n}\right|^{a}}
$$

is increasing in $r$, for $r \in(0,1)$. Moreover, $N(r)$ is constant in $\left(r_{1}, r_{2}\right)$ if and only if $u$ is homogeneous of degree $N\left(r_{1}\right)$ in $B_{r_{2}} \backslash B_{r_{1}}$.

Following the proof of Wei01, Lemma 4.1] and using Lemma4.10 and Lemma4.11 we have the corollary below:

Corollary 4.12. Let $u$ be a-harmonic in $B_{1}$, even in $x_{n}$, with $u(0)=0$. If $a<0$ assume also that $\left|\nabla_{x^{\prime}} u(0)\right|=0$. Then

$$
\int_{B_{r}}|\nabla u|^{2}\left|x_{n}\right|^{a} \geq C(a) \int_{\partial B_{r}} u^{2}\left|x_{n}\right|^{a}
$$

where $C(a)=1$ if $a \geq 0$ and $C(a)=2$ if $a<0$. Moreover, equality holds if and only if $u$ is homogeneous of degree $C(a)$.

Proof. We first prove the inequality by contradiction. If the statement is false then there is $r_{0} \in(0,1)$ such that $N\left(r_{0}\right)<C(a)$. By Lemma 4.11, this implies $N(r)<C(a)$ for all $r \in\left(0, r_{0}\right)$. Let

$$
w_{r}(x)=\frac{w(r x)}{\left(\int_{\partial B_{1}} w^{2}(r x)\left|x_{n}\right|^{a}\right)^{\frac{1}{2}}} .
$$

Then

$$
\int_{\partial B_{1}} w_{r}^{2}\left|x_{n}\right|^{a}=1
$$

$w_{r}$ is bounded in $W^{1,2}\left(B_{1},\left|x_{n}\right|^{a}\right)$ and $a$-harmonic in $B_{1}$. By the compactness of the trace operator, Lemma 4.8 and Corollary 4.6, we can find a sequence $r_{j} \rightarrow 0+$ such that $w_{r_{j}}$ converges strongly in $L^{2}\left(\partial B_{1},\left|x_{n}\right|^{a}\right) \cap W^{1,2}\left(B_{1 / 2},\left|x_{n}\right|^{a}\right) \cap C\left(B_{1 / 2}\right)$ (and also in $C_{x^{\prime}}^{1}\left(B_{1 / 2}\right)$ if $a<0$ from Lemma 4.9) to a limit function $w_{0}$. Hence, for $s \in(0,1 / 2)$ there holds

$$
N(0+, w)=\lim _{r_{j} \rightarrow 0} N\left(s r_{j}, w\right)=\lim _{r_{j} \rightarrow 0} N\left(s, w_{r_{j}}\right)=N\left(s, w_{0}\right),
$$

so that $N\left(s, w_{0}\right)$ is constant. Therefore, by Lemma 4.11, $w_{0}$ is homogeneous of degree $N(0+, w)<C(a)$ in $B_{1 / 2}$. We can extend $w_{0}$ to $a$-homogeneous function in whole $\mathbb{R}^{n}$. Note that $a$-harmonic functions are real analytic off the thin space $\mathbb{R}^{n-1} \times\{0\}$, and therefore $a$-harmonic continuations are unique.

Now, if $a \geq 0$, Lemma 4.10 implies that $w_{0}=0$ contradicting (4.6).

If $a<0$, then Lemma4.10 implies that $w_{0}$ is linear. Due to the extra assumption when $a<0, w_{0}=0$ and a contradiction is reached again.

Finally, if equality holds for some $r_{0} \in(0,1)$, then it must hold for all $r<r_{0}$, so by Lemma 4.11 and unique continuation, $u$ must be homogeneous of degree $C(a)$. 
The next result establishes a Weiss-type monotonicity formula, which will be an important tool in our study. Since the proof is rather technical, for readers' convenience we have moved it to the appendix.

Theorem 4.13 (Weiss-type monotonicity formula). Let $u$ be a minimizer of (4.1). Then the functional

$$
\begin{aligned}
r \mapsto W(r)=W(r, u):= & \frac{1}{r^{n-a}} \int_{B_{r}}|\nabla u|^{2}\left|x_{n}\right|^{a}+\frac{4}{r^{n-a}} \int_{B_{r}^{\prime}}\left(\lambda_{+} u^{+}+\lambda_{-} u^{-}\right) \\
& -\frac{1-a}{r^{n+1-a}} \int_{\partial B_{r}} u^{2}\left|x_{n}\right|^{a}
\end{aligned}
$$

is nondecreasing for $0<r<1$. Moreover, $W$ is constant for $r \in\left(r_{1}, r_{2}\right)$ if and only if $u$ is homogeneous of degree $1-a$ in the ring $r_{1}<|x|<r_{2}$.

As we will see, the case $a=0$ will require a special treatment. The following result, known as the Alt-Caffarelli-Friedman (ACF) monotonicity formula, will be instrumental in the study of that case.

Lemma 4.14 (ACF monotonicity formula). Let $\left\{w_{+}, w_{-}\right\}$be a pair of nonnegative continuous subharmonic functions in $B_{R}$ such that $w_{+} \cdot w_{-}=0$ in $B_{R}$. Then the functional

$$
r \mapsto \Phi\left(r, w_{+}, w_{-}\right):=\frac{1}{r^{4}} \int_{B_{r}} \frac{\left|\nabla w_{+}\right|^{2}}{|x|^{n-2}} \int_{B_{r}} \frac{\left|\nabla w_{-}\right|^{2}}{|x|^{n-2}}
$$

is finite and nondecreasing for $0<r<R$.

For the proof of Lemma 4.14 we refer to [CS05, PSU12, or the original paper ACF 84. We also note that we can change the center of the ball to $x_{0}$ by replacing $\Phi$ with $\Phi^{x_{0}}$ given by

$$
\Phi^{x_{0}}\left(r, w_{+}, w_{-}\right):=\frac{1}{r^{4}} \int_{B_{r}\left(x_{0}\right)} \frac{\left|\nabla w_{+}\right|^{2}}{\left|x-x_{0}\right|^{n-2}} \int_{B_{r}\left(x_{0}\right)} \frac{\left|\nabla w_{-}\right|^{2}}{\left|x-x_{0}\right|^{n-2}}
$$

Remark 4.15. For $w_{r}(x):=w(r x) / r$, the functional $\Phi(r)$ enjoys the following rescaling property:

$$
\Phi\left(r, w_{+}, w_{-}\right)=\Phi\left(1,\left(w_{+}\right)_{r},\left(w_{-}\right)_{r}\right)
$$

Remark 4.16. If $u$ is a minimizer of (4.1) and $a=0$, then $w_{ \pm}=u^{ \pm}$are subharmonic so that Lemma 4.14 applies to the pair.

\section{Regularity of Minimizers}

In this section we prove the Hölder continuity of the minimizers of (4.1) for all $a \in(-1,1)$ and $C^{1, \alpha}$ regularity along the thin space when $a \in(-1,0)$. As we will see these regularity results are not optimal, but necessary for technical reasons. The optimal regularity is established in the next section.

Theorem 5.1 (Hölder continuity). Let $u$ be a minimizer of (4.1). Then $u \in$ $C^{0, s}\left(B_{1}\right), s=(1-a) / 2$. Moreover, there exists $C=C\left(a, n, \lambda_{ \pm}\right)>0$ such that

$$
\|u\|_{C^{0, s}\left(B_{1 / 2}\right)} \leq C\|u\|_{L^{2}\left(B_{1},\left|x_{n}\right|^{a}\right)} .
$$


Proof. The proof is almost identical with the proof of Theorem 3.1 in All12, we spell out the details below.

By Corollary 4.7, we know that $u$ is bounded in $B_{3 / 4}$. Then put

$$
C_{0}=4 \max \left\{\lambda_{+}, \lambda_{-}\right\} \sup _{B_{3 / 4}}|u| .
$$

For $r \in(0,3 / 4)$, let $v$ be the function that is $a$-harmonic in $B_{r}$ and equals $u$ on $\partial B_{r}$ (in other words, $v$ is the $a$-harmonic replacement of $u$ on $B_{r}$ ). Then since $u$ minimizes $J_{a}$ on all balls inside $B_{1}$, there holds

$$
\int_{B_{r}}\left(|\nabla u|^{2}-|\nabla v|^{2}\right)\left|x_{n}\right|^{a} \leq 4 \int_{B_{r}^{\prime}}\left(\lambda_{+} v^{+}+\lambda_{-} v^{-}\right) \leq C_{0} r^{n-1},
$$

due to the estimate

$$
\sup _{B_{r}}|v| \leq \sup _{B_{r}}|u| .
$$

Since $u=v$ on $\partial B_{r}$ we also have

$$
\int_{B_{r}} \nabla v \cdot(\nabla u-\nabla v)\left|x_{n}\right|^{a}=0
$$

which implies

$$
\int_{B_{r}}\left(|\nabla u|^{2}-|\nabla v|^{2}\right)\left|x_{n}\right|^{a}=\int_{B_{r}}|\nabla u-\nabla v|^{2}\left|x_{n}\right|^{a},
$$

so that (5.1) becomes

$$
\int_{B_{r}}|\nabla u-\nabla v|^{2}\left|x_{n}\right|^{a} \leq C_{0} r^{n-1} .
$$

This can now be iterated in a standard way (cf. Theorem 3.1 in [All12]), to imply

$$
\int_{B_{r}}|\nabla u|^{2}\left|x_{n}\right|^{a} \leq C r^{n-1}
$$

for $0<r<3 / 4$, where $C$ might also depend on the $W^{1,2}\left(B_{3 / 4},\left|x_{n}\right|^{a}\right)$-norm of $u$, which by the energy inequality (see Corollary 4.6) can be estimated in terms of $L^{2}\left(B_{1},\left|x_{n}\right|^{a}\right)$-norm of $u$. By Hölder's inequality this gives

$$
\int_{B_{r}}|\nabla u| \mathrm{d} x \leq\left\||\nabla u| x_{n}^{a / 2}\right\|_{L^{2}\left(B_{r}\right)}\left\|x_{n}^{-a / 2}\right\|_{L^{2}\left(B_{r}\right)} \leq C r^{n-(1+a) / 2} .
$$

The estimate above is valid for all balls $B_{r}\left(x^{\prime}, 0\right)$ centered at the thin space, as long as they are contained in $B_{3 / 4}$. From arguments used in the proof of Morrey's embedding this yields the estimates

$$
\left|u\left(x^{\prime}, 0\right)-f_{B_{r}\left(x^{\prime}, 0\right)} u\right| \leq C r^{s}, \quad\left|u\left(x^{\prime}, 0\right)-u\left(y^{\prime}, 0\right)\right| \leq C r^{s},
$$

where $s=(1-a) / 2$. Then, arguing as at the end of the proof of Theorem 3.1 in All12 we can conclude that $u$ is $s$-Hölder continuous in $B_{1 / 2}$ with the required estimate on the $C^{0, s}$ norm.

Remark 5.2. The fact that $u$ is a minimizer of $J_{a}$ in $B_{1}$ can be changed with the following conditions:

(1) $L_{a} u=0$ in $B_{1}^{+}$; 
(2) $\left|\lim _{x_{n} \rightarrow 0+} x_{n}^{a} u_{x_{n}}\left(x^{\prime}, x_{n}\right)\right| \leq \mu$ for $x^{\prime} \in B_{1}^{\prime}$ in the sense that

$$
\left|\int_{B_{1}^{+}}(\nabla u \cdot \nabla \psi) x_{n}^{a}\right| \leq \mu \int_{B_{1}^{\prime}}|\psi|,
$$

for any $\psi \in C_{0}^{\infty}\left(B_{1}\right)$ (cf. Lemma 4.1);

(3) $|u| \leq M$ in $B_{1}^{+}$.

Then we will have that

$$
\|u\|_{C^{0, s}\left(B_{1 / 2}\right)} \leq C(M, \mu, n, a) .
$$

Instead of (5.1) one will have to use

$$
\begin{aligned}
\left.\left|\int_{B_{r}} \nabla u(\nabla v-\nabla u)\right| x_{n}\right|^{a} \mid & \leq 2 \mu\left|\int_{B_{1}^{\prime}}\right| u-v|| \\
& \leq C(M, \mu, n) r^{n-1}
\end{aligned}
$$

together with

$$
\int_{B_{r}} \nabla v(\nabla v-\nabla u)\left|x_{n}\right|^{a}=0 .
$$

We now turn to the $C^{1, \alpha}$ regularity along the thin space when $a \in(0,1)$.

Theorem $5.3\left(C^{1, \alpha}\right.$ regularity, $\left.a<0\right)$. Let $u$ be minimizer of (4.1) in $B_{1}$ with $|u| \leq M$ in $B_{1}$. If $a \in(-1,0)$, then $u \in C^{1, \alpha}\left(B_{1}^{\prime}\right)$ for $\alpha<-a$. Moreover,

$$
\|u\|_{C^{1, \alpha}\left(B_{1 / 2}^{\prime}\right)} \leq C(M, n, a, \alpha)\left(\|u\|_{L^{2}\left(B_{1},\left|x_{n}\right|^{a}\right)}+\max \left\{\lambda_{+}, \lambda_{-}\right\}\right)
$$

Proof. Let $f$ be defined as in Lemma 4.1 We extend $f$ to all of $\mathbb{R}^{n-1}$ by defining $f\left(y^{\prime}\right)=0$ if $y^{\prime} \notin B_{1}$. Then $f \in L^{\infty}\left(\mathbb{R}^{n-1}\right)$ with compact support, so we can use the Riesz potential

$$
I_{2 s} f\left(x^{\prime}\right)=c_{n, s} \int_{\mathbb{R}^{n-1}} \frac{f\left(y^{\prime}\right) \mathrm{d} y^{\prime}}{\left|x^{\prime}-y^{\prime}\right|^{n-2 s}}
$$

as the inverse to the fractional Laplacian in the sense that $\left(-\Delta_{x^{\prime}}\right)^{s} I_{2 s} f=f$. Since $a<0$, by Proposition 2.9 in [Sil07], $I_{2 s} f \in C^{1, \alpha}\left(\mathbb{R}^{n-1}\right)$ for any $\alpha<-a$. We now use the extension operator as in [CS07] on $I_{2 s} f$ to obtain an $a$-harmonic function $v$ in the upper half plane with Dirichlet data $I_{2 s} f$, so

$$
\left.v\right|_{\mathbb{R}^{n-1}}=I_{2 s} f \text {. }
$$

We may evenly reflect $v$ across the thin space. If $w=u-v$, then for all $\psi \in C_{0}^{2}\left(B_{1}\right)$

$$
\int_{B_{1}^{\prime}}\left|x_{n}\right|^{a} \nabla w \nabla \psi=0 .
$$

Then $L_{a} w=0$ in all of $B_{1}$. In particular, $w$ is $C^{\infty}$ regular on the thin ball $B_{1}^{\prime}$ in any $x^{\prime}$ direction (cf. Lemma 4.9). Since $u=v+w$, it follows that

$$
u \in C^{1, \alpha}\left(B_{1}^{\prime}\right) \quad \text { for } \alpha<-a .
$$

The interior estimate on $u$ follows by the interior estimate on $v$ (see Proposition 2.9 in [Sil07]) and for the $a$-harmonic function $w$ (see Lemma 4.9).

Remark 5.4. Similarly to Remark 5.2 , in Theorem 5.3 we may change the requirement that $u$ is a minimizer of $J_{a}$ in $B_{1}$ with conditions (1)-(3) in Remark 5.2 Then, the conclusion will be that

$$
\|u\|_{C^{1, \alpha}\left(B_{1 / 2}^{\prime}\right)} \leq C(M, \mu, a, \alpha, n)
$$


for any $0<\alpha<-a$.

\section{Optimal Growth AND REgularity}

Now we have all the tools needed to prove the optimal growth of order $1-a$, which follows below.

Theorem 6.1 (Optimal growth). Let $u$ be a bounded minimizer of (4.1), with $|u| \leq M$ in $B_{1}$. Then the following holds.

(i) If $a \geq 0$, then

$$
\sup _{B_{r}\left(x^{\prime}, 0\right)}\left|u(y)-u\left(x^{\prime}, 0\right)\right| \leq C r^{1-a}
$$

for $0<r<1 / 2$ and $x^{\prime} \in B_{1 / 2}^{\prime}$, with $C=C\left(a, M, \lambda_{ \pm}, n\right)$.

(ii) If $a<0$, then

$$
\begin{aligned}
& \sup _{B_{r}\left(x^{\prime}, 0\right)}\left|u(y)-u\left(x^{\prime}, 0\right)-\left(y-\left(x^{\prime}, 0\right)\right) \cdot \nabla_{x^{\prime}} u\left(x^{\prime}, 0\right)\right| \leq C r^{1-a}, \\
& \text { for } 0<r<1 / 2 \text { and } x^{\prime} \in B_{1 / 2}^{\prime} \text {, with } C=C\left(a, M, \lambda_{ \pm}, n\right) .
\end{aligned}
$$

Proof. Case i: $a \geq 0$. For $0<r<1 / 2$ and $x^{\prime} \in B_{1 / 2}^{\prime}$ let

$$
S_{r}\left(u, x^{\prime}\right)=\sup _{B_{r}}\left|u-u\left(x^{\prime}, 0\right)\right| .
$$

If the assertion does not hold then we can find a sequence of minimizers $u_{j}$, points $x_{j}^{\prime} \in B_{1 / 2}^{\prime}$ and radii $r_{j} \rightarrow 0$ such that

$$
\frac{S_{r_{j}}\left(u_{j}, x_{j}^{\prime}\right)}{r_{j}^{1-a}}=C_{j} \rightarrow \infty,
$$

and

$$
S_{r_{j} 2^{k}}\left(u_{j}, x_{j}^{\prime}\right) \leq 2^{k(1-a)} S_{r_{j}}\left(u_{j}, x_{j}^{\prime}\right),
$$

for all nonnegative integers $k$ such that $2^{k} r_{j} \leq 1$. Let now

$$
v_{j}(x)=\frac{u_{j}\left(r_{j} x+\left(x_{j}^{\prime}, 0\right)\right)-u_{j}\left(x_{j}^{\prime}, 0\right)}{C_{j} r_{j}^{1-a}} .
$$

Then

(1) $v_{j}$ is even in $x_{n}$-variable

(2) $v_{j}$ satisfies $L_{a} v_{j}=0$ in $B_{R}^{+}$for $R<1 / r_{j}$;

(3) For any $\psi \in C_{0}^{\infty}\left(B_{R}\right)$

$$
\int_{B_{R}^{+}}\left(\nabla v_{j} \cdot \nabla \psi\right) x_{n}^{a} \leq \frac{\max \left\{\lambda_{+}, \lambda_{-}\right\}}{C_{j}} \int_{B_{R}^{\prime}}|\psi| ;
$$

(4) $\sup _{B_{2^{k}}}\left|v_{j}\right| \leq 2^{k(1-a)}$, whenever $2^{k} r_{j} \leq 1$;

(5) $v_{j}(0)=0$;

(6) $\sup _{B_{1}}\left|v_{j}\right|=1$.

By Theorem 5.1 and Remark 5.2, $v_{j}$ is uniformly bounded in $C^{0, s}\left(B_{R}\right)$ for all $R<1 / r_{j}$. Passing to the limit, we obtain that $v_{j}$ converges (over a subsequence) to a function $v_{0} \in C^{0, s}\left(\mathbb{R}^{n}\right)$ which satisfies

$$
\int_{\mathbb{R}^{n}}\left(\nabla v_{0} \cdot \nabla \psi\right)\left|x_{n}\right|^{a}=0
$$


for any $\psi \in C_{0}^{\infty}\left(\mathbb{R}^{n}\right)$. In other words, $v_{0}$ is $a$-harmonic in $\mathbb{R}^{n}$, even in $x_{n}$-variable. Besides, we will have that

$$
\left|v_{0}(x)\right| \leq C\left(1+|x|^{1-a}\right), \quad v_{0}(0)=0, \quad \sup _{B_{1}}\left|v_{0}\right|=1 .
$$

The Liouville-type theorem in Lemma 4.10 now implies implies that $v_{0}$ is a polynomial.

At this point we need to split the proof into two different cases depending on $a$.

Subcase i.a: $a>0$. Since the growth at infinity is less than linear $v_{0}$ must be constant and thus $v_{0} \equiv 0$, which is clearly a contradiction with the properties of $v_{0}$ above.

Subcase i.b: $a=0$. Again, due to the growth condition, we can deduce that $v_{0}$ is linear. To get a contradiction, we intend to use the ACF monotonicity formula, but in order to do so, we need to make the following observation. For the sequences $u_{j}, x_{j}^{\prime}$ and $r_{j}$ in our contradictory assumption, without loss of generality we may assume that the following holds:

$(\alpha)$ either $u_{j}\left(x_{j}^{\prime}, 0\right)=0$ for all $j$,

( $\beta) B_{r_{j}}^{\prime}\left(x_{j}^{\prime}\right) \subset\left\{u_{j}>0\right\}$ for all $j$, or

$(\gamma) B_{r_{j}}^{\prime}\left(x_{j}^{\prime}\right) \subset\left\{u_{j}<0\right\}$ for all $j$.

Indeed, in the case when $x_{j}^{\prime} \notin \Gamma_{u_{j}}$ and $r_{j}<\operatorname{dist}\left(x_{j}^{\prime}, \Gamma_{u_{j}}\right)$, we may simply replace $x_{j}^{\prime}$ with its closest point $y_{j}^{\prime} \in \Gamma_{u_{j}}$ and $r_{j}$ with $2 r_{j}$ and convert it to case $(\alpha)$.

Cases $(\alpha)-(\gamma)$ translate into the following ones for functions $v_{j}$.

$\left(\alpha^{\prime}\right) v_{j}(0,0)=0$ for all $j$,

$\left(\beta^{\prime}\right) v_{j}>0$ in $B_{1}^{\prime}$ for all $j$,

$\left(\gamma^{\prime}\right) v_{j}<0$ in $B_{1}^{\prime}$ for all $j$.

In the cases $\left(\beta^{\prime}\right)$ and $\left(\gamma^{\prime}\right)$ we obtain immediately that the limit $v_{0}$ has a sign in $B_{1}^{\prime}$. Since we also know that $v_{0}$ is linear and even in $x_{n}$, this immediately implies that $v_{0}$ must be identically zero, a contradiction with property (6) of $v_{j}$. In the case $\left(\alpha^{\prime}\right)$, which is the same as $(\alpha)$, we observe that we have the additional property that

$$
\Delta\left(u_{j}^{ \pm}\right) \geq 0 \text { in } B_{1},
$$

see Lemma 4.5. Then we can apply the ACF monotonicity formula (Lemma 4.14). We will have that for any $s>0$ and large $j$

$$
\begin{aligned}
\Phi\left(s, v_{j}^{+}, v_{j}^{-}\right) & =\frac{1}{C_{j}^{2}} \Phi^{x_{j}}\left(s r_{j}, u_{j}^{+}, u_{j}^{-}\right) \\
& \leq \frac{1}{C_{j}^{2}} \Phi^{x_{j}}\left(1 / 4, u_{j}^{+}, u_{j}^{-}\right) \leq \frac{C(n)}{C_{j}^{2}}\left\|u_{j}\right\|_{L^{2}\left(B_{1}\right)}^{2} \rightarrow 0 .
\end{aligned}
$$

Hence, $\Phi\left(s, v_{0}^{+}, v_{0}^{-}\right)=0$ for $s>0$, and we can conclude that either $v_{0}^{+}=0$ or $v_{0}^{-}=0$. Since $v_{0}$ is linear and even in $x_{n}$, we again conclude that $v_{0}$ is identically zero, which contradicts property (6) of $v_{j}$.

Case ii: $a<0$. Here we define instead

$$
S_{r}\left(u, x^{\prime}\right)=\sup _{y \in B_{r}\left(x^{\prime}, 0\right)}\left|u(y)-u\left(x^{\prime}, 0\right)-y^{\prime} \cdot \nabla_{x^{\prime}} u\left(x^{\prime}, 0\right)\right| .
$$


If the assertion does not hold then we can find a sequence of minimizers $u_{j}$, points $x_{j}^{\prime} \in B_{1 / 2}^{\prime}$ and a radii $r_{j} \rightarrow 0$ such that

$$
\frac{S_{r_{j}}\left(u_{j}, x_{j}^{\prime}\right)}{r_{j}^{1-a}}=C_{j} \rightarrow \infty
$$

and

$$
S_{r_{j} 2^{k}}\left(u_{j}, x_{j}^{\prime}\right) \leq 2^{k(1-a)} S_{r_{j}}\left(u_{j}, x_{j}^{\prime}\right),
$$

for all nonnegative integers $k$ such that $2^{k} r_{j} \leq 1$. Let now

$$
v_{j}(x)=\frac{u_{j}\left(r_{j} x+\left(x^{\prime}, 0\right)\right)-u_{j}\left(x^{\prime}, 0\right)-\left(r_{j} x^{\prime}\right) \cdot \nabla_{x^{\prime}} u_{j}\left(x_{j}^{\prime}, 0\right)}{C_{j} r_{j}^{1-a}} .
$$

Then, again as before,

(1) $v_{j}$ is even in $x_{n}$-variable

(2) $v_{j}$ satisfies $L_{a} v_{j}=0$ in $B_{R}^{+}$for $R<1 / r_{j}$;

(3) For any $\psi \in C_{0}^{\infty}\left(B_{R}\right)$

$$
\int_{B_{R}^{+}}\left(\nabla v_{j} \cdot \nabla \psi\right) x_{n}^{a} \leq \frac{\max \left\{\lambda_{+}, \lambda_{-}\right\}}{C_{j}} \int_{B_{R}^{\prime}}|\psi| ;
$$

(4) $\sup _{B_{2 k}}\left|v_{j}\right| \leq 2^{k(1-a)}$, whenever $2^{k} r_{j} \leq 1$;

(5) $v_{j}(0)=\left|\nabla_{x^{\prime}} v_{j}(0)\right|=0$;

(6) $\sup _{B_{1}}\left|v_{j}\right|=1$.

By Theorem 5.1, Remark 5.2, Theorem 5.3 and Remark 5.4 there is a subsequence, again labelled $v_{j}$, converging in $C_{\mathrm{loc}}^{0, s} \cap C_{\mathrm{loc}}^{1, \alpha}\left(\mathbb{R}^{n-1} \times 0\right)$ and then by (3) above also weakly in $W_{\text {loc }}^{1,2}\left(\left|x_{n}\right|^{a}\right)$ to a limit function $v_{0}$. By passing to the limit in (3) and (4) above, we see that

$$
L_{a} v_{0}=0 \quad \text { in } \mathbb{R}^{n}
$$

and

$$
\left|v_{0}(x)\right| \leq C\left(1+|x|^{1-a}\right), \quad v_{0}(0)=\left|\nabla_{x^{\prime}} v_{0}(0)\right|=0, \quad \sup _{B_{1}}\left|v_{0}\right|=1 .
$$

Since $v_{0}$ is also even in $x_{n}$ variable, Lemma 4.10 now implies that $v_{0}$ is a polynomial. Since the growth at infinity is less than quadratic, $v_{0}$ must be linear. The properties of $v_{0}$ above imply that $v_{0} \equiv 0$, a contradiction.

Remark 6.2. As in Remark 5.2 and 5.4, the assertions of Theorem 6.1 hold true when the requirement that $u$ is a minimizer of $J_{a}$ in $B_{1}$ is replaced by conditions (1)-(2) in Remark [5.2, for $a \neq 0$. The only change in the conclusion would be that now $C=C(a, M, \mu, n)$. The result is not true for $a=0$ as the example $u=\operatorname{Re} z \ln z$ shows. The lacking information is the fact that for minimizers, $u^{ \pm}$are subsolutions.

With the proposition above, we can now prove the optimal regularity. More precisely, we prove the following version of Theorem 1.2

Theorem 6.3 (Optimal regularity). Let $u$ be a bounded minimizer of (4.1), with $0 \in \Gamma^{+} \cup \Gamma^{-}$and $|u| \leq M$ in $B_{1}$. Then the following holds.

(i) If $a \geq 0$, then $u \in C^{0,1-a}\left(B_{1 / 4}\right)$ with

$$
\|u\|_{C^{0,1-a}\left(B_{1 / 4}\right)} \leq C\left(a, M, \lambda_{ \pm}, n\right)
$$


(ii) If $a<0$, then $u \in C^{1,-a}\left(B_{1 / 4}^{ \pm} \cup B_{1 / 4}^{\prime}\right)$ with

$$
\|u\|_{C^{1,-a}\left(B_{1 / 4}^{ \pm} \cup B_{1 / 4}^{\prime}\right)} \leq C\left(a, M, \lambda_{ \pm}, n\right) .
$$

Proof. Case i: $a \geq 0$. First, form the growth estimate in Theorem 6.1 and Lemma 4.9 applied in the ball $B_{x_{n}}\left(x^{\prime}, x_{n}\right)$, we obtain that

$$
\left|\nabla_{x^{\prime}} u(x)\right| \leq C x_{n}^{-a}, \quad \text { for } x \in B_{1 / 4}^{+},
$$

where $C=C\left(a, M, \lambda_{ \pm}, n\right)$. Besides, we claim that a similar estimate holds also in $x_{n}$-direction:

$$
\left|u_{x_{n}}(x)\right| \leq C x_{n}^{-a}, \quad \text { for } x \in B_{1 / 4}^{+} .
$$

To show this estimate, we note that from Lemma4.1 we know that the function $\tilde{u}=$ $x_{n}^{a} u_{x_{n}}$ is uniformly bounded in $B_{1 / 2}^{\prime}$, with the bound depending on $\lambda^{ \pm}$. Moreover, it is known that $L_{-a} \tilde{u}=0$ in $B_{1}^{+}$(see for instance CS07 for an explanation). Next, let $v$ be the bounded solution of

$$
L_{-a} v=0 \quad \text { in } \mathbb{R}_{+}^{n}, \quad v\left(x^{\prime}, 0\right)=\tilde{u}\left(x^{\prime}, 0\right) \zeta\left(x^{\prime}\right), \quad x^{\prime} \in \mathbb{R}^{n-1},
$$

with some cutoff function $\zeta \in C_{0}^{\infty}\left(B_{1}^{\prime}\right)$ which equals 1 on $B_{1 / 2}^{\prime}$. Then, by using the Poisson formula (see [CS07]), we will have

$$
\|v\|_{L^{\infty}\left(B_{1 / 2}^{+}\right)} \leq C\|\tilde{u} \zeta\|_{L^{\infty}\left(B_{1 / 2}^{\prime}\right)} \leq C\left(a, M, \lambda^{ \pm}, n\right) .
$$

Furthermore, let $w=u-v$ and extend $w$ in an odd manner across the thin space. Then, $w$ is $(-a)$-harmonic in $B_{1 / 2}$. Hence, from Lemma 4.5 and Corollaries 4.6 and 4.7, we have

$$
\begin{aligned}
\|w\|_{L^{\infty}\left(B_{1 / 2}^{+}\right)} & \leq C\|w\|_{L^{2}\left(B_{3 / 4},\left|x_{n}\right|^{-a}\right)} \\
& =C\|\nabla u\|_{L^{2}\left(B_{3 / 4},\left|x_{n}\right|^{a}\right)} \leq C \sup _{B_{1}}|u|=C M .
\end{aligned}
$$

Combining the estimates for $v$ and $w$, we obtain a similar estimate for $\tilde{u}$, which implies (6.2).

With estimates (6.1) -(6.2), we can now prove the Hölder continuity of $u$. Let $x, y \in B_{1 / 4}^{+} \cup B_{1 / 4}^{\prime}$ and without loss of generality assume that $x_{n} \leq y_{n}$. Consider the following possibilities:

(1) $|x-y| \geq y_{n} / 2$. In this case, by Theorem 6.1, we have

$$
\begin{aligned}
|u(x)-u(y)| & \leq\left|u\left(x^{\prime}, 0\right)-u\left(y^{\prime}, 0\right)\right|+\left|u(x)-u\left(x^{\prime}, 0\right)\right|+\left|u(y)-u\left(y^{\prime}, 0\right)\right| \\
& \leq C\left|x^{\prime}-y^{\prime}\right|^{1-a}+C x_{n}^{1-a}+C y_{n}^{1-a} \leq C|x-y|^{1-a} .
\end{aligned}
$$

(2) $|x-y|<y_{n} / 2$. In this case, $x, y \in B_{y_{n} / 2}(y)$ and we have that by (6.1) -6.2)

$$
|\nabla u| \leq C y_{n}^{-a} \text { in } B_{y_{n} / 2}(y) .
$$

Hence

$$
|u(x)-u(y)| \leq C y_{n}^{-a}|x-y| \leq C|x-y|^{1-a} .
$$

Thus, we obtain the desired Hölder continuity of $u$ in $B_{1 / 4}^{+} \cup B_{1 / 4}^{\prime}$. By symmetry in $x_{n}$ variable, we therefore have the same Hölder continuity in $B_{1 / 4}$.

Case ii: $a<0$. First, note that the estimate (6.2) still holds in this case. To prove the desired $C^{1,-a}$ regularity we will obtain similar estimates for the second derivatives. 
To this end, take a point $x=\left(x^{\prime}, x_{n}\right) \in B_{1 / 4}^{+}$. Then $\tilde{u}=x_{n}^{a} u_{x_{n}}$ is $(-a)$-harmonic in $B_{x_{n}}(x)$ and applying Lemma 4.9 we obtain

$$
\left|\nabla_{x^{\prime}} u_{x_{n}}(x)\right| \leq C x_{n}^{-1-a} \text { for } x \in B_{1 / 4}^{+} .
$$

Further, using that $w(y)=u(y)-u\left(x^{\prime}, 0\right)-\left(y-\left(x^{\prime}, 0\right)\right) \cdot \nabla_{x^{\prime}} u\left(x^{\prime}, 0\right)$, is $a$-harmonic in $B_{x_{n}}(x)$, we obtain by Lemma 4.9

$$
\left|D_{x^{\prime}}^{2} u(x)\right|=\left|D_{x^{\prime}}^{2} w(x)\right| \leq \frac{C}{x_{n}^{2}} \sup _{B_{x_{n} / 2}(x)}|w| \leq C x_{n}^{-1-a}, \quad \text { for } x \in B_{1 / 4}^{+},
$$

where the last inequality follows from the optimal growth estimate in Theorem 6.1 . Now, using the equation for $u$, we conclude that

$$
\left|u_{x_{n} x_{n}}(x)\right|=\left|-\Delta_{x^{\prime}} u(x)-a x_{n}^{-1} u_{x_{n}}(x)\right| \leq C x_{n}^{-1-a}, \quad \text { for } x \in B_{1 / 4}^{+} .
$$

Combining (6.3)-6.5), we arrive at

$$
\left|D^{2} u(x)\right| \leq C x_{n}^{-1-a}, \quad \text { for } x \in B_{1 / 4}^{+} .
$$

Now taking $x, y \in B_{1 / 4}^{+} \cup B_{1 / 4}^{\prime}$ and repeating the the arguments as at the end of Case i, we readily conclude that

$$
|\nabla u(x)-\nabla u(y)| \leq C|x-y|^{-a} .
$$

(In the estimate of $u_{x_{n}}$ we use that $u_{x_{n}}$ is identically zero on $B_{1 / 2}^{\prime}$, by estimate (6.2).) Furthermore, using Theorem 5.3 integrating (6.3) from $\left(x^{\prime}, 0\right)$ to $\left(x^{\prime}, x_{n}\right)$, and using (6.2) one more time, we also establish that

$$
|\nabla u| \leq C \quad \text { for } x \in B_{1 / 4}^{+}
$$

Hence,

$$
\|u\|_{C^{1,-a}\left(B_{1 / 4}^{+} \cup B_{1 / 4}^{\prime}\right)} \leq C\left(a, M, \lambda^{ \pm}, n\right),
$$

as required.

The proof of Theorem 1.2 is now immediate.

Proof of Theorem 1.2. The proof follows from the local boundedness (see Corollary 4.7), Theorem 6.3, and a simple covering argument by balls.

From Remarks 5.2, 5.4 and 6.2, we also have the following version of Theorem 6.3 , when $a \neq 0$.

Theorem 6.4. Let $a \neq 0$. Assume

(1) $L_{a} u=0$ in $B_{1}^{+}$;

(2) $\left|\lim _{x_{n} \rightarrow 0+} x_{n}^{a} u_{x_{n}}\left(x^{\prime}, x_{n}\right)\right| \leq \mu$ for $x^{\prime} \in B_{1}^{\prime}$ in the sense that

$$
\left|\int_{B_{1}^{+}}(\nabla u \cdot \nabla \psi) x_{n}^{a}\right| \leq \mu \int_{B_{1}^{\prime}}|\psi|
$$

for any $\psi \in C_{0}^{\infty}\left(B_{1}\right)$

(3) $|u| \leq M$ in $B_{1}^{+}$.

Then the following holds:

(i) If $a>0$, then $u \in C^{0,1-a}\left(B_{1 / 4}^{+}\right)$with

$$
\|u\|_{C^{0,1-a}\left(B_{1 / 4}^{+}\right)} \leq C(a, M, \mu, n) .
$$


(ii) If $a<0$, then $u \in C^{1,-a}\left(B_{1 / 4}^{+} \cup B_{1 / 4}^{\prime}\right)$ with

$$
\|u\|_{C^{1,-a}\left(B_{1 / 4}^{+} \cup B_{1 / 4}^{\prime}\right)} \leq C(a, M, \mu, n) .
$$

\section{Nondegeneracy}

In this section we prove a certain nondegeneracy property for the minimizers that will be instrumental for the proof of our second main result, Theorem 1.3. We follow the outline as given in $\mathrm{AP} 12$.

Theorem 7.1 (Nondegeneracy). Fix $0<t<1$, and let $u$ be a minimizer of (4.1). There exists $\epsilon>0$ with $\epsilon$ depending only on $\lambda_{ \pm}$and $t$, such that if $\left.u\right|_{\partial B_{r}} \leq \epsilon r^{1-a}$ $\left(\left.u\right|_{\partial B_{r}} \geq-\epsilon r^{1-a}\right)$ then

$$
u(x) \leq 0 \quad(u(x) \geq 0) \quad \text { for } x \in B_{t r}^{\prime} .
$$

We will need the following lemma, which is along the lines of Corollary 4.4 .

Lemma 7.2. Let $u$ be a minimizer of (4.1) such that $\left.u\right|_{\partial B_{1}}=M$. Then $u$ is symmetric about the line $\left(0, \ldots, 0, x_{n}\right)$ and

$$
u\left(x^{\prime}, 0\right)=f\left(\left|x^{\prime}\right|\right)
$$

where $f$ is a nondecreasing function. Therefore, if the coincidence set is nonempty then the coincidence set $\{u=0\}=\bar{B}_{\rho}^{\prime}$ for some $0 \leq \rho \leq 1$.

Proof. Extend $u$ to be a function on the cube $Q$ with side length 2, by defining $u(x)=M$ for $x \notin B_{1}$. We now apply Steiner symmetrization (as defined in Kaw85, page 82]) to the function $w=M-u$ on lines parallel to $\mathbb{R}^{n-1} \times\{0\}$. If we only consider $\left\{x|| x_{n} \mid>\epsilon\right\}$, then $w$ is Lipschitz. Then by [Kaw85, page 82], if we Steiner symmetrize $w$ to obtain $v$ deduce for each $\epsilon>0$ :

$$
\int_{B_{1} \cap\left\{\left|x_{n}\right|>\epsilon\right\}}|\nabla u|^{2}\left|x_{n}\right|^{a}=\int_{B_{1} \cap\left\{\left|x_{n}\right|>\epsilon\right\}}|\nabla w|^{2}\left|x_{n}\right|^{a} \geq \int_{B_{1} \cap\left\{\left|x_{n}\right|>\epsilon\right\}}|\nabla v|^{2}\left|x_{n}\right|^{a}
$$

$v$ will have the same boundary values as $w$ on $\partial B_{1}$. Then by letting $\epsilon \rightarrow 0$ we obtain

$$
\int_{B_{1}}|\nabla u|^{2}\left|x_{n}\right|^{a}=\int_{B_{1}}|\nabla w|^{2}\left|x_{n}\right|^{a} \geq \int_{B_{1}}|\nabla v|^{2}\left|x_{n}\right|^{a} .
$$

Finally, we note that

$$
\int_{B_{1}^{\prime}} \lambda_{+} u
$$

is invariant under Steiner symmetrization. Since minimizers are unique, we see that our minimizer is Steiner symmetric about the line $\left(0, \ldots, 0, x_{n}\right)$ and $\{u=0\}$ is connected and centered at the origin.

Lemma 7.3. Let $u_{\epsilon}$ be the minimizer of (4.1) subject to the boundary conditions $u_{\epsilon} \equiv \epsilon$ on $\partial B_{1}$. Then $u_{\epsilon} \equiv 0$ on $B_{\rho}^{\prime}$ for some $\rho=\rho(\epsilon)>0$. Furthermore $\rho(\epsilon) \rightarrow 1$ as $\epsilon \rightarrow 0$.

Proof. It is clear that the minimizer $u_{\epsilon} \geq 0$. From the comparison principle $u_{\epsilon_{1}} \leq$ $u_{\epsilon_{2}}$ if $\epsilon_{1} \leq \epsilon_{2}$. Suppose by way of contradiction that $u_{\epsilon}\left(x^{\prime}, 0\right)>0$ for all $\epsilon$ and for $x^{\prime}$ in the ring

$$
\mathcal{R}=\left\{\left(x^{\prime}, 0\right) \in B_{1}^{\prime}\left|0 \leq r_{1}<\right| x^{\prime} \mid<r_{2} \leq 1\right\} .
$$


By Lemma 4.1

$$
\lim _{t \rightarrow 0} t^{a} \frac{\partial u_{\epsilon}}{\partial x_{n}}\left(x^{\prime}, t\right)=\lambda_{+}
$$

Thus

$$
u_{\epsilon}-\frac{\lambda_{+}}{1-a}\left|x_{n}\right|^{1-a}
$$

will be $a$-harmonic in the tube

$$
\mathcal{T}=\left\{x \in B_{1}\left|0 \leq r_{1}<\right| x^{\prime} \mid<r_{2} \leq 1\right\} .
$$

Now, $u_{\epsilon} \rightarrow u \equiv 0$ uniformly, which implies that $-\frac{\lambda_{+}}{1-a}\left|x_{n}\right|^{1-a}$ is $a$-harmonic in $\mathcal{T}$. This is clearly a contradiction. Thus, there exists $\epsilon$ and $y^{\prime} \in \mathcal{R}$ such that $u_{\epsilon}\left(y^{\prime}, 0\right)=0$. Then, by Lemma 7.2, $u_{\epsilon} \equiv 0$ on $B_{r_{1}}^{\prime}$.

We are now able to prove the nondegeneracy result.

Proof of Theorem 7.1. First we note that by rescaling we only need to prove Theorem 7.1 on the unit ball $B_{1}$. Pick $0<t<1$. Lemma 7.3 proves that there exists $\epsilon$ depending on $t$ and $\lambda_{+}$such that $\left\{u_{\epsilon}=0\right\}=B_{t}^{\prime}$. By the comparison principle if $u$ is a minimizer such that $u \leq u_{\epsilon}=\epsilon$ on $\partial B_{1}$, then $u \leq 0$ on $B_{t}^{\prime}$. The case for which $u \geq-\epsilon$ is proven similarly.

Theorem 7.1 immediately implies the corollary below, which is the result we will be using later on.

Corollary 7.4. If $u$ is a minimizer and $0 \in \Gamma^{+}\left(0 \in \Gamma^{-}\right)$, then

$$
\sup _{\partial B_{r}} u \geq C r^{1-a} \quad\left(\inf _{\partial B_{r}} u \leq-C r^{1-a}\right),
$$

where $C$ depends only on $a, \lambda_{ \pm}$and $n$.

\section{Blowups AND GLOBAl SOlutions}

In this section we study the blowups and global solutions that will be the main step towards proving Theorem 1.3 .

Let $u$ be a minimizer of (4.1) and assume that $0 \in \Gamma^{+} \cup \Gamma^{-}$. For $r>0$ consider the rescalings

$$
u_{r}(x)=\frac{u(r x)}{r^{1-a}}, \quad x \in B_{1 / r} .
$$

From the scaling properties of $J_{a}$, it is easy to see that $u_{r}$ is a minimizer of $J_{a}$ in $B_{1 / r}$. By the optimal growth estimate we will then have that

$$
\sup _{B_{R}}\left|u_{r}\right| \leq C R^{1-a}, \quad(r<1 / R)
$$

when $a \geq 0$, so the family $\left\{u_{r}\right\}_{0<r<1}$ is locally bounded in $\mathbb{R}^{n}$. Note that to have the same conclusion when $a<0$ we must assume additionally that $\left|\nabla_{x^{\prime}} u(0)\right|=0$. The local boundedness implies boundedness in $W_{\text {loc }}^{1,2}\left(\mathbb{R}^{n},\left|x_{n}\right|^{a}\right)$, by the energy inequality. Thus, over a sequence $r_{k} \rightarrow 0$ the rescalings $u_{r_{k}}$ will converge to a certain $u_{0}$ weakly in $W_{\text {loc }}^{1,2}\left(\mathbb{R}^{n},\left|x_{n}\right|^{a}\right)$. (This convergence is actually strong, as we prove in Lemma 8.1 below.) Passing to a subsequence we may assume that the convergence is strong in $L_{\text {loc }}^{2}\left(\mathbb{R}^{n},\left|x_{n}\right|^{a}\right)$ and $L_{\text {loc }}^{2}\left(\mathbb{R}^{n-1} \times\{0\}\right)$. We call such $u_{0}$ a blowup of $u$ at the origin. A standard argument also shows that the blowup $u_{0}$ will be a minimizer of $J_{a}$ on any $U \Subset \mathbb{R}^{n}$. Such minimizer we will call global minimizers of $J_{a}$. 
Our analysis of blowups starts with the following lemma on the strong convergence of minimizers.

Lemma 8.1 (Strong convergence). Let $\left\{u_{k}\right\}$ be a sequence of minimizers of $J_{a}$ in $D^{+}$that converges weakly in $W^{1,2}\left(D^{+}, x_{n}^{a}\right)$ to some minimizer $u$. Then, over a subsequence, $u_{k}$ converges strongly to $u$ in $W^{1,2}\left(U^{+}, x_{n}^{a}\right)$ for any $U \Subset D$.

Proof. Take a test function $\eta \in C_{0}^{\infty}(D)$ such that

$$
0 \leq \eta \leq 1, \quad \eta \equiv 1 \text { in a neighborhood of } \bar{U} .
$$

Then, integrating by parts we have

$$
\begin{aligned}
\int_{D^{+}}\left|\nabla\left(u_{k}-u\right)\right|^{2} \eta^{2}\left|x_{n}\right|^{a}= & \int_{D^{\prime}}\left(f_{k}-f\right)\left(u_{k}-u\right) \eta^{2} \\
& -2 \int_{D^{+}}\left(u_{k}-u\right) \eta \nabla \eta \nabla\left(u_{k}-u\right)\left|x_{n}\right|^{a},
\end{aligned}
$$

where $f_{k}\left(x^{\prime}\right)=-\lim _{x_{n} \rightarrow 0+} x_{n}^{a} \partial_{x_{n}} u_{k}\left(x^{\prime}, x_{n}\right)$ and $f\left(x^{\prime}\right)=-\lim _{x_{n} \rightarrow 0+} x_{n}^{a} \partial_{x_{n}} u\left(x^{\prime}, x_{n}\right)$ in the sense of Lemma 4.1, which also tells that $f_{k}$ and $f$ are uniformly bounded by $\max \left\{\lambda_{ \pm}\right\}$. Using that bound and applying the Young's inequality to the second integral on the right-hand side in the usual manner, we obtain that

$$
\int_{U^{+}}\left|\nabla\left(u_{k}-u\right)\right|^{2}\left|x_{n}\right|^{a} \leq C \int_{D^{\prime} \cap K}\left|u_{k}-u\right|+C \int_{D^{+} \cap K}\left(u_{k}-u\right)^{2}\left|x_{n}\right|^{a},
$$

where $K=\operatorname{supp} \eta \Subset D$. The proof now follows from the strong convergence of $u_{k}$ to $u$ in $L^{2}\left(D^{\prime} \cap K\right)$ and $L^{2}\left(D^{+} \cap K,\left|x_{n}\right|^{a}\right)$ by the compactness of the trace operator and the Sobolev embedding.

Having the strong convergence of minimizers combined with the Weiss-type monotonicity formula, we immediately obtain the following property of blowups.

Lemma 8.2. Let $u$ be a minimizer of (4.1) with $0 \in \Gamma^{+} \cup \Gamma^{-}$. If a $<0$ make the further assumption that $\left|\nabla_{x^{\prime}} u(0)\right|=0$. Suppose also that $u_{r_{k}}$ converges to a blowup $u_{0}$. Then $u_{0}$ is homogeneous of degree $(1-a)$.

Proof. We will use the following scaling property of the Weiss energy functional

$$
W(\rho r, u)=W\left(\rho, u_{r}\right)
$$

Now, by Lemma 8.1. we may assume that $u_{r_{k}} \rightarrow u_{0}$ strongly in $W^{1,2}\left(U,\left|x_{n}\right|^{a}\right)$ for any $U \Subset \mathbb{R}^{n}$ and we can pass in the limit in the Weiss energy functional to obtain

$$
W\left(\rho, u_{0}\right)=\lim _{k \rightarrow \infty} W\left(\rho, u_{r_{k}}\right)=\lim _{k \rightarrow \infty} W\left(\rho r_{k}, u\right)=W(0+, u)
$$

for any $\rho>0$. This implies that $W\left(\cdot, u_{0}\right) \equiv$ const $=W(0+, u)$ and consequently that $u_{0}$ is homogeneous of degree $(1-a)$, by the second part of Theorem 4.13 .

Lemma 8.3 (Homogeneous global solutions). Let $a \geq 0$ and let $u$ be a global minimizer of $J_{a}$ and assume $u$ is homogeneous of degree $(1-a)$. Then $u=c\left|x_{n}\right|^{1-a}$ for some constant $c$.

Proof. Choose $c$ to be such that if $v=u-c\left|x_{n}\right|^{1-a}$, then

$$
\int_{\partial B_{1}}\left|x_{n}\right|^{a} v=0
$$


We claim that

$$
\frac{-\lambda_{-}}{(1-a)} \leq c \leq \frac{\lambda_{+}}{(1-a)}
$$

The heuristic idea behind the claim is that if $c$ is too large then $L_{a} v \leq 0$ in all of $B_{1}$, and so $v(0)>0$. To proceed proving this claim we use the definition of $f$ in as given in Lemma 4.1. Suppose that $c>\lambda_{+} /(1-a)$. Using that $\operatorname{div}\left(\left|x_{n}\right|^{a} \nabla v\right)=0$ off the thin spaces, we integrate by parts to obtain

$$
\int_{\partial B_{r}}\left|x_{n}\right|^{a} v_{\nu}=2 \int_{B_{r}^{\prime}}(f-c(1-a))<0,
$$

since $f \leq \lambda_{+} \leq c(1-a)$. Also, for almost every $r$

$$
\frac{d}{d r}\left[\frac{1}{r^{n-1+a}} \int_{\partial B_{r}}\left|x_{n}\right|^{a} v\right]=\int_{\partial B_{r}}\left|x_{n}\right|^{a} v_{\nu}
$$

It follows that

$$
\frac{1}{r^{n-1+a}} \int_{\partial B_{r}}\left|x_{n}\right|^{a} v \quad \text { is decreasing in } r .
$$

Then $v(0)>0$. This is a contradiction since $u(0)=0$, and consequently $v(0)=0$.

A similar argument shows that $c \geq-\lambda_{-} /(1-a)$.

Now we use integration by parts to obtain

$$
\int_{B_{r}}\left|x_{n}\right|^{a}|\nabla v|^{2}=\int_{\partial B_{r}}\left|x_{n}\right|^{a} v v_{\nu}-2 \int_{B_{r}^{\prime}}(f-c(1-a)) v .
$$

By homogeneity of $v$ we obtain that $v_{\nu}=(1-a) v / r$. We now turn to simplifying the integral along the thin space. Note that $v=u$ on the thin space. Also by Lemma 4.1. we may write $f\left(y^{\prime}\right)$ explicitly when $u\left(y^{\prime}, 0\right) \neq 0$. Since we are multiplying by $u$ on the thin space we only need to consider the case when $u\left(y^{\prime}, 0\right) \neq 0$. So our equality above becomes

$$
\begin{aligned}
\int_{B_{r}}\left|x_{n}\right|^{a}|\nabla v|^{2}= & \frac{1-a}{r} \int_{\partial B_{r}}\left|x_{n}\right|^{a} v^{2} \\
& -2 \int_{B_{r}^{\prime}}\left(\lambda_{+}-c(1-a)\right) u^{+}+\left(\lambda_{-}+c(1-a)\right) u^{-}
\end{aligned}
$$

We then define $c_{+}=\left(\lambda_{+}-c(1-a)\right) / 2$ and $c_{-}=\left(\lambda_{-}+c(1-a)\right) / 2$. Now $c_{ \pm} \geq 0$ by our claim above. Also either $c_{+}>0$ or $c_{-}>0$. If we move everything to the left hand side in (8.3) and multiply by $r$, we obtain

$$
r\left(\int_{B_{r}}\left|x_{n}\right|^{a}|\nabla v|^{2}+4 \int_{B_{r}^{\prime}}\left(c_{+} v^{+}+c_{-} v^{-}\right)\right)-(1-a) \int_{\partial B_{r}}\left|x_{n}\right|^{a} v^{2}=0 .
$$

If $w$ is the $a$-harmonic replacement of $v$ in $B_{r}$, then $w(0)=0$ by (8.1) and $w$ is even in $x_{n}$ since $v$ is even. Now suppose $v$ is not equivalently zero. From Corollary 4.12 we obtain

$$
1 \leq N(w, r) \leq N(v, r) .
$$

Combining the above inequality with (8.4) we have

$$
1 \leq N(w, r) \leq N(v, r) \leq r \frac{\int_{B_{r}}\left|x_{n}\right|^{a}|\nabla v|^{2}+4 \int_{B_{r}^{\prime}}\left(c_{+} v^{+}+c_{-} v^{-}\right)}{\int_{\partial B_{r}}\left|x_{n}\right|^{a} v^{2}}=1-a .
$$


If $a>0$, then we obtain an immediate contradiction, and thus for $a>0, u \equiv$ $c\left|x_{n}\right|^{1-a}$. If $a=0$, then necessarily

$$
\int_{B_{r}^{\prime}}\left(c_{+} v^{+}+c_{-} v^{-}\right)=0
$$

Then $v\left(x^{\prime}, 0\right) \equiv 0$. By using an odd reflection in $x_{n}$ variable, we obtain a harmonic function, homogeneous of degree 1 in all of $\mathbb{R}^{n}$. Thus, by the Liouville theorem we conclude that $v=c_{3} x_{n}$ if $x_{n}>0$ and by even symmetry then $v=c_{3}\left|x_{n}\right|$ for all $x$. By (8.1) we must have $c_{3}=0$ and so $v \equiv 0$.

We conclude this section with the following generalization of Lemma 8.3, which will be important in the next section when studying the limits of rescalings of possibly different functions, when we don't have the homogeneity, but can control the growth at infinity.

Lemma 8.4 (Global minimizers). Let $u$ be a global minimizer of $J_{a}$ such that $0 \in \Gamma^{+} \cup \Gamma^{-}$for $a \geq 0$. Assume also that

$$
|u(x)| \leq C\left(1+|x|^{1-a}\right) .
$$

Then $u=c\left|x_{n}\right|^{1-a}$ for some constant $c$.

Proof. We first perform a blow-up $u_{r_{j}} \rightarrow u_{0}$, and conclude by Lemma 8.3 that $u_{0}=c_{0}\left|x_{n}\right|^{1-a}$. Next, considering again the usual rescalings

$$
u_{R}(x)=\frac{u(R x)}{R^{1-a}}
$$

and noticing that $u_{R}$ are also locally uniformly bounded as $R \rightarrow \infty$, we can extract a subsequence $R_{j} \rightarrow \infty$ such that $u_{R_{j}} \rightarrow u_{\infty}$. Arguing precisely as in Lemma 8.2 we obtain that $u_{\infty}$ is homogeneous and thus, by Lemma 8.3, $u_{\infty}=c_{\infty}\left|x_{n}\right|^{1-a}$.

On the other hand, we can compute that $W\left(r,\left|x_{n}\right|^{1-a}\right) \equiv 0$, which implies

$$
0=W\left(1, u_{0}\right)=\lim _{r \rightarrow 0} W(r, u) \leq W(s, u) \leq \lim _{R \rightarrow \infty} W(R, u)=W\left(1, u_{\infty}\right)=0,
$$

for any $s>0$. Hence, $W(u, s) \equiv 0$ and $u$ is homogeneous, and again by Lemma 8.3 . $u=c\left|x_{n}\right|^{1-a}$.

\section{Structure of the Free boundary}

In this section we prove Theorem 1.3 in the following more expanded form.

Theorem 9.1 (Structure of the free boundary). Let $a \geq 0$ and let $u$ be $a$ bounded minimizer of (4.1) such that $|u| \leq M$ in $B_{1}$. Then

$$
\Gamma^{+} \cap \Gamma^{-}=\emptyset \text {. }
$$

More specifically, there is $c_{0}=c\left(a, M, \lambda_{ \pm}, n\right)>0$ such that if $x_{0} \in \Gamma^{+} \cap B_{1 / 2}^{\prime}$ then $B_{c_{0}}^{\prime}\left(x_{0}\right) \cap \Gamma^{-}=\emptyset$.

Proof. Let $x_{0} \in \Gamma_{u}^{+} \cap B_{1 / 2}$. By translation and rescaling, we may assume that $x_{0}=0$. Then, if the statement of the theorem fails, then there exists a sequence of minimizers $u_{j}$ of (4.1) satisfying

$$
\left|u_{j}\right| \leq M, \quad 0 \in \Gamma_{u_{j}}^{+}, \quad\left|\nabla_{x^{\prime}} u_{j}(0)\right|=0 \quad \text { if } a<0 .
$$


together with a sequence of points $x^{j} \in \Gamma_{u_{j}}^{-}$such that $r_{j}=\left|x^{j}\right| \rightarrow 0$. We will show that this is impossible. Let

$$
v_{j}(x)=\left(u_{j}\right)_{r_{j}}(x)=\frac{u_{j}\left(r_{j} x\right)}{r_{j}^{1-a}} .
$$

By Theorem 5.1 combined with Theorem 6.1 $\left\{v_{j}\right\}$ is uniformly bounded in $C^{\alpha}\left(B_{R}\right) \cap$ $W^{1,2}\left(B_{R},\left|x_{n}\right|^{a}\right)$ for every ball $B_{R}$. Moreover, we know:

(1) $v_{j}$ is a minimizer of $J_{a}$ in $B_{R} \subset B_{1 / r_{j}}$.

(2) By Theorem 6.1 we have the growth estimates

$$
\left|v_{j}(x)\right| \leq C|x|^{1-a}, \quad|x| \leq 1 /\left(2 r_{j}\right) .
$$

(3) Since $0 \in \Gamma_{v_{j}}^{+}$and $z^{j}=x^{j} / r_{j} \in \Gamma_{v_{j}}^{-} \cap B_{1}^{\prime}$, by Theorem 7.1 we have

$$
\sup _{B_{t}} v_{j} \geq C t^{1-a}, \quad \inf _{B_{t}\left(z^{j}\right)} v_{j} \leq-C t^{1-a} .
$$

Consequently, we can extract a subsequence, again labeled $v_{j}$, converging locally uniformly to $v_{0}$, a global minimizer of $J_{a}$. Due to the nondegeneracy property (3) above, we also know that $v_{0}$ has nontrivial positive and negative phases. On the other hand, by (2) we also know that

$$
\left|v_{0}(x)\right| \leq C|x|^{1-a}, \quad \text { for any } x \in \mathbb{R}^{n}
$$

and thus from Lemma 8.4 we conclude that $v_{0}=c\left|x_{n}\right|^{1-a}$. Clearly, this is not possible.

Theorem 1.3 now follows.

Proof of Theorem 1.3. This is an immediate consequence of the local boundedness (see Corollary 4.7), Theorem 9.1, and a simple covering argument by balls.

We conclude this section and the paper by providing an example showing that when $a<0$ it is possible for the two phases to meet at a point where the thin gradient vanishes. This is done by constructing a minimizer whose Weiss energy at a point is negative.

Example 9.2 (The two phases can meet where the gradient vanishes, when $a<0$ ). For simplicity in our construction we will assume $\lambda_{+}=\lambda_{-}=\lambda$. Fix some $i \in$ $\{1, \ldots, n-1\}$ and let $\ell(x)=M x_{i}$. Then consider the minimizer of the functional $J_{a}$ with boundary values given by $u=\ell$ on $\partial B_{1}$. We claim that there is an $M>0$ such that $0 \in \Gamma^{+} \cap \Gamma^{-}$and $\nabla_{x^{\prime}} u(0)=0$. By symmetry of the boundary values of $u$ on $\partial B_{1}$ combined with our choice of $\lambda_{+}=\lambda_{-}$and the fact that minimizers are unique, it is clear that $u(0)=0$. Now we look at the Weiss energy of $\ell$ in $B_{1}$. By homogeneity of $\ell$

$$
W(1, \ell)=M \int_{B_{1}^{\prime}} \lambda\left|x_{i}\right|+a M^{2} \int_{\partial B_{1}} x_{i}^{2}\left|x_{n}\right|^{a},
$$

and we see that for large enough $M$ (since $a<0), W(1, \ell)<0$. Since $u$ and $\ell$ coincide on $\partial B_{1}$, this implies that $W(1, u)<0$. Now we argue that $0 \in \Gamma_{u}^{+} \cup \Gamma_{u}^{-}$. Indeed, otherwise then $u \equiv 0$ in $B_{\rho}^{\prime}$ for some small $\rho$ (recall $u(0)=0$ ). If we reflect $u$ by odd reflection across the thin space we obtain $\tilde{u}$ which is $a$-harmonic in the solid ball $B_{\rho}$ so that $W(\rho, u)=W(\rho, \tilde{u})$. By Corollary 4.12, $W(\rho, \tilde{u}) \geq 0$, contradicting $W(\rho, u) \leq W(1, u)<0$. Thus, $0 \in \Gamma_{u}^{+} \cup \Gamma_{u}^{-}$and by symmetry and uniqueness, $0 \in \Gamma_{u}^{+} \cap \Gamma_{u}^{-}$. 
If $\left|\nabla_{x^{\prime}} u(0)\right|=0$, then the claim is proved. If not, $\left|\nabla_{x^{\prime}} u(0)\right| \neq 0$ for $M$ large enough. In this case let

$$
\bar{M}=\inf \left\{M:\left|\nabla_{x^{\prime}} u(0)\right| \neq 0\right\} .
$$

Non-degeneracy (cf. Theorem [7.1) implies $u \equiv 0$ near the origin for $M$ small enough. Hence, $\left|\nabla_{x^{\prime}} u(0)\right|=0$ for $M$ small enough and thus $\bar{M}>0$. Interior $C^{1, \alpha_{-}}$ convergence (cf. Theorem 6.3) implies $\left|\nabla_{x^{\prime}} u_{\bar{M}}(0)\right|=0$. We claim that $0 \in \Gamma_{u_{\bar{M}}}$. Suppose towards a contradiction that $0 \notin \Gamma_{u_{\bar{M}}}$.

By symmetry and uniqueness of minimizers, $u(0)=0$ and $0 \in \Gamma_{u}^{+}\left(0 \in \Gamma_{u}^{-}\right)$ implies $0 \in \Gamma_{u}^{-}\left(0 \in \Gamma_{u}^{+}\right)$. For $M>\bar{M}$, the fact that $0 \in \Gamma_{u}^{ \pm}$implies

$$
\sup _{\partial B_{r}} u \geq C r^{1-a}, \quad \inf _{\partial B_{r}} u \leq-C r^{1-a}
$$

for some $C>0$ and for $0<r<1$. By the uniform convergence, this holds true also for $u_{\bar{M}}$. If $0 \notin \Gamma_{u_{\bar{M}}}$ then $u_{\bar{M}} \equiv 0$ in $B_{\rho}^{\prime}$ for some $\rho>0$. Now we perform a blowup at the origin of $u \bar{M}$ and obtain $u_{0}$, which is homogeneous of degree $1-a$, by Lemma 8.2. By the scaling invariance, $u_{0}$ satisfies (9.1). Since $u \equiv 0$ in $B_{\rho}^{\prime}$ for some $\rho>0$, then $u_{0} \equiv 0$ in $\mathbb{R}^{n-1}$. If we extend $u_{0}$ by odd reflection in the $x_{n}$-variable, we obtain an $a$-harmonic function in the whole space. Since $u_{0}$ is homogeneous of degree $1-a$, we must have $u_{0}=c\left|x_{n}\right|^{1-a}$ (cf. Lemma 4.9 for instance), contradicting (9.1).

\section{Appendix A. Proof of Weiss monotonicity formula}

Proof of Theorem 4.13. The proof is very similar to that of Theorem 4.3 in All12] and we omit the details that are derived identically.

Using that $u$ is a minimizer of (4.1), we obtain that for a.e. $r \in(0,1)$ we have the equality

$$
\begin{aligned}
0=( & n-2+a) \int_{B_{r}}\left|x_{n}\right|^{a}|\nabla u|^{2}-r \int_{\partial B_{r}}\left|x_{n}\right|^{a}\left(|\nabla u|^{2}-2 u_{\nu}^{2}\right) \\
& +4(n-1) \int_{B_{r}^{\prime}}\left(\lambda_{+} u^{+}+\lambda_{-} u^{-}\right)-4 r \int_{\partial B_{r}^{\prime}}\left(\lambda_{+} u^{+}+\lambda_{-} u^{-}\right) \\
= & (n-1) \int_{B_{r}}\left|x_{n}\right|^{a}|\nabla u|^{2}-r \int_{\partial B_{r}}\left|x_{n}\right|^{a}|\nabla u|^{2} \\
& +4(n-1) \int_{B_{r}^{\prime}}\left(\lambda_{+} u^{+}+\lambda_{-} u^{-}\right)-4 r \int_{\partial B_{r}^{\prime}}\left(\lambda_{+} u^{+}+\lambda_{-} u^{-}\right) \\
& -(1-a) \int_{B_{r}}\left|x_{n}\right|^{a}|\nabla u|^{2}+2 r \int_{\partial B_{r}}\left|x_{n}\right|^{a} u_{\nu}^{2} .
\end{aligned}
$$

By Lemma 4.1.

$$
\int_{B_{r}}\left|x_{n}\right|^{a}|\nabla u|^{2}=\int_{\partial B_{r}}\left|x_{n}\right|^{a} u u_{\nu}+2 \int_{B_{r}^{\prime}}\left(\lambda_{+} u^{+}+\lambda_{-} u^{-}\right)
$$


and hence

$$
\begin{aligned}
0=(n & -a) \int_{B_{r}}\left|x_{n}\right|^{a}|\nabla u|^{2}-r \int_{\partial B_{r}}\left|x_{n}\right|^{a}|\nabla u|^{2} \\
& +4(n-a) \int_{B_{r}^{\prime}}\left(\lambda_{+} u^{+}+\lambda_{-} u^{-}\right)-4 r \int_{\partial B_{r}^{\prime}}\left(\lambda_{+} u^{+}+\lambda_{-} u^{-}\right) \\
& -2(1-a) \int_{\partial B_{r}}\left|x_{n}\right|^{a} u \cdot u_{\nu}+2 r \int_{\partial B_{r}}\left|x_{n}\right|^{a} u_{\nu}^{2}
\end{aligned}
$$

Now multiply both sides of the equation by $-r^{-n+1-a}$ to obtain that for a.e. $r \in$ $(0,1)$

$$
\begin{aligned}
0= & {\left[\frac{1}{r^{n-a}} \int_{B_{r}}\left|x_{n}\right|^{a}|\nabla u|^{2}\right]^{\prime}+\left[\frac{4}{r^{n-a}} \int_{B_{r}^{\prime}}\left(\lambda_{+} u^{+}+\lambda_{-} u^{-}\right)\right]^{\prime} } \\
& -\frac{2}{r^{n-a}} \int_{\partial B_{r}}\left|x_{n}\right|^{a}\left(\frac{(1-a) u u_{\nu}}{r}-u_{\nu}^{2}\right) .
\end{aligned}
$$

As in [All12, we also have that for almost every $r$

$$
\text { (A.2) } \frac{\mathrm{d}}{\mathrm{d} r}\left[\frac{1-a}{r^{n+1-a}} \int_{\partial B_{r}}\left|x_{n}\right|^{a} u^{2}\right]=\frac{2}{r^{n-a}} \int_{\partial B_{r}}\left|x_{n}\right|^{a}\left(\frac{(1-a) u u_{\nu}}{r}-\frac{(1-a)^{2} u^{2}}{r^{2}}\right) \text {. }
$$

We then add and subtract the piece from (A.2) to obtain for almost every $r$

$$
\begin{aligned}
0= & {\left[\frac{1}{r^{n-a}} \int_{B_{r}}\left|x_{n}\right|^{a}|\nabla u|^{2}\right]^{\prime}+\left[\frac{4}{r^{n-a}} \int_{B_{r}^{\prime}}\left(\lambda_{+} u^{+}+\lambda_{-} u^{-}\right)\right]^{\prime} } \\
& -\left[\frac{1-a}{r^{n+1-a}} \int_{\partial B_{r}}\left|x_{n}\right|^{a} u^{2}\right]^{\prime}-\frac{2}{r^{n-a}} \int_{\partial B_{r}}\left|x_{n}\right|^{a}\left(\frac{(1-a) u}{r}-u_{\nu}\right)^{2}
\end{aligned}
$$

Thus, $W^{\prime} \geq 0$, and $W^{\prime}=0$ on the interval $r_{1}<r<r_{2}$ if and only if $u$ is homogeneous of degree $2 s=(1-a)$ on the ring $r_{1}<|x|<r_{2}$.

\section{REFERENCES}

[AC04] I. Athanasopoulos and L. A. Caffarelli, Optimal regularity of lower dimensional obstacle problems, Zap. Nauchn. Sem. S.-Peterburg. Otdel. Mat. Inst. Steklov. (POMI) 310 (2004), no. Kraev. Zadachi Mat. Fiz. i Smezh. Vopr. Teor. Funkts. 35 [34], 49-66, 226.

[ACF84] Hans Wilhelm Alt, Luis A. Caffarelli, and Avner Friedman, Variational problems with two phases and their free boundaries, Trans. Amer. Math. Soc. 282 (1984), no. 2, 431461. MR 732100 (85h:49014)

[ACS08] I. Athanasopoulos, L. A. Caffarelli, and S. Salsa, The structure of the free boundary for lower dimensional obstacle problems, Amer. J. Math. 130 (2008), no. 2, 485-498.

[All12] Mark Allen, Separation of a lower-dimensional free boundary in a two phase problem, Math. Res. Lett (2012), 19pp., to appear.

[AP12] Mark Allen and Arshak Petrosyan, A two-phase problem with a lower-dimensional free boundary, Interfaces Free Bound. 14 (2012), no. 3, 307-342.

[CS05] Luis Caffarelli and Sandro Salsa, A geometric approach to free boundary problems, Graduate Studies in Mathematics, vol. 68, American Mathematical Society, Providence, RI, 2005. MR 2145284 (2006k:35310)

[CS07] Luis Caffarelli and Luis Silvestre, An extension problem related to the fractional Laplacian, Comm. Partial Differential Equations 32 (2007), no. 7-9, 1245-1260.

[CSS08] Luis A. Caffarelli, Sandro Salsa, and Luis Silvestre, Regularity estimates for the solution and the free boundary of the obstacle problem for the fractional Laplacian, Invent. Math. 171 (2008), no. 2, 425-461. 
[DL76] G. Duvaut and J.-L. Lions, Inequalities in mechanics and physics, Springer-Verlag, Berlin, 1976, Translated from the French by C. W. John, Grundlehren der Mathematischen Wissenschaften, 219.

[FKS82] Eugene B. Fabes, Carlos E. Kenig, and Raul P. Serapioni, The local regularity of solutions of degenerate elliptic equations, Comm. Partial Differential Equations 7 (1982), no. $1,77-116$

[GP09] Nicola Garofalo and Arshak Petrosyan, Some new monotonicity formulas and the singular set in the lower dimensional obstacle problem, Invent. Math. 177 (2009), no. 2, 415-461.

[Kaw85] Bernhard Kawohl, Rearrangements and convexity of level sets in PDE, Lecture Notes in Mathematics, vol. 1150, Springer-Verlag, Berlin, 1985.

[Kil97] T. Kilpeläinen, Smooth approximation in weighted Sobolev spaces, Comment. Math. Univ. Carolin. 38 (1997), no. 1, 29-35. MR 1455468 (98g:46043)

[Nek93] Aleš Nekvinda, Characterization of traces of the weighted Sobolev space $W^{1, p}\left(\Omega, d_{M}^{\epsilon}\right)$ on $M$, Czechoslovak Math. J. 43(118) (1993), no. 4, 695-711. MR 1258430 (95i:46041)

[PSU12] Arshak Petrosyan, Henrik Shahgholian, and Nina Uraltseva, Regularity of free boundaries in obstacle-type problems, Graduate Studies in Mathematics, vol. 136, American Mathematical Society, Providence, RI, 2012.

[Sil07] Luis Silvestre, Regularity of the obstacle problem for a fractional power of the Laplace operator, Comm. Pure Appl. Math. 60 (2007), no. 1, 67-112.

[SUW04] Henrik Shahgholian, Nina Uraltseva, and Georg S. Weiss, Global solutions of an obstacle-problem-like equation with two phases, Monatsh. Math. 142 (2004), no. 1-2, 27-34. MR 2065019 (2005c:35301)

[SUW07] _ The two-phase membrane problem—regularity of the free boundaries in higher dimensions, Int. Math. Res. Not. IMRN (2007), no. 8, Art. ID rnm026, 16. MR 2340105 (2009b:35444)

[SW06] Henrik Shahgholian and Georg S. Weiss, The two-phase membrane problem-an intersection-comparison approach to the regularity at branch points, Adv. Math. 205 (2006), no. 2, 487-503. MR 2258264 (2007k:35167)

[Ura01] N. N. Uraltseva, Two-phase obstacle problem, J. Math. Sci. (New York) 106 (2001), no. 3, 3073-3077, Function theory and phase transitions.

[Wei01] G. S. Weiss, An obstacle-problem-like equation with two phases: pointwise regularity of the solution and an estimate of the Hausdorff dimension of the free boundary, Interfaces Free Bound. 3 (2001), no. 2, 121-128.

Department of Mathematics, The University of Texas at Austin, Austin, TX 78712, USA

E-mail address: mallen@math.utexas.edu

Department of Mathematics, Royal Institute of Technology, 10044 Stockholm, SweDEN

E-mail address: eriklin@kth.se

Department of Mathematics, Purdue University, West Lafayette, in 47907, USA

E-mail address: arshak@math.purdue.edu 